\title{
Computing Bounds for Second Zagreb Coindex of Sum Graphs
}

\author{
Muhammad Javaid (D), ${ }^{1}$ Muhammad Ibraheem ${ }^{(D)},{ }^{1}$ Uzma Ahmad ${ }^{\circ},{ }^{2}$ and Q. Zhu ${ }^{3,4}$ \\ ${ }^{1}$ Department of Mathematics, School of Science, University of Management and Technology, Lahore 54770, Pakistan \\ ${ }^{2}$ Department of Mathematics, University of the Punjab, Lahore, Pakistan \\ ${ }^{3}$ School of Mathematics and Statistics, Hunan Normal University, Changsha, Hunan 4100081, China \\ ${ }^{4}$ Department of Mathematics, School of Information Science and Engineering, Chengdu University, Chengdu 610106, China \\ Correspondence should be addressed to Muhammad Javaid; javaidmath@gmail.com
}

Received 23 April 2021; Accepted 1 June 2021; Published 16 July 2021

Academic Editor: Stylianos Georgantzinos

Copyright (c) 2021 Muhammad Javaid et al. This is an open access article distributed under the Creative Commons Attribution License, which permits unrestricted use, distribution, and reproduction in any medium, provided the original work is properly cited.

\begin{abstract}
Topological indices or coindices are one of the graph-theoretic tools which are widely used to study the different structural and chemical properties of the under study networks or graphs in the subject of computer science and chemistry, respectively. For these investigations, the operations of graphs always played an important role for the study of the complex networks under the various topological indices or coindices. In this paper, we determine bounds for the second Zagreb coindex of a well-known family of graphs called $F$-sum ( $S$-sum, $R$-sum, $Q$-sum, and $T$-sum) graphs in the form of Zagreb indices and coindices of their factor graphs, where these graphs are obtained by using four subdivision-related operations and Cartesian product of graphs. At the end, we illustrate the obtained results by providing the exact and bonded values of some specific $F$-sum graphs.
\end{abstract}

\section{Introduction}

A topological index (TI) is a function from the set of graphs to the set of real numbers that assigns the different numerical values to the different graphs unless the graphs are isomorphic. Moreover, TIs are essential tools to discuss various physical and chemical properties of the graphs such as volume, density, connectivity, boiling point, freezing point, and heat of formation and evaporation $[1,2]$. TIs are also used to study the quantitative structure property relationships (QSPRs), quantitative structure activity relationships (QSARs), and clinical practices of various medications in the subject of cheminformatics and pharmaceutical industries, respectively (see [3-5]). Mainly TIs have three types such as degree, distance, and polynomial based but the degree-based TIs are more studied than others (see the most recent review [6]).

Firstly, an American Chemist Harry Wiener (1947) used a distance-based TI to calculate the boiling point of paraffin (see [7]). First and second Zagreb indices are introduced by Gutman and Trinajsti in 1972; these indices are used to calculate total $\pi$-electron energy of alternant hydrocarbons [8]. Kinkar and Gutman calculated different relations between the second Zagreb index of a graph and its complement (see [9]). Yan et al. computed sharp bounds for the second Zagreb index of different unicyclic graphs [10]. Carlos et al. calculated the second Zagreb index of the graphs with minimum and maximum vertex degrees. They also investigated trees with the maximum value of the second Zagreb index among all trees with maximum vertex degree [11].

Recently, Zagreb coindices are introduced by Ashrafi et al., and they studied them for the derived graphs obtained by the operations of joining, union, disjunction, Cartesian product, and corona product (see $[12,13])$. Kinkar et al. calculated the first Zagreb index and multiplicative Zagreb coindices of tree (see [14]). Gutman obtained coindices of graphs and their complements (see [15]). Nilanjan et al. calculated $F$-coindex of some graph operations (see [16]). Javaid et al. calculated the first Zagreb connection index and coindex of some derived graphs [17]. Ramane et al. calculated coindices for the transmission and reciprocal 
transmission-based graphs (see [18]). Mansour and Song computed $a$ and $(a, b)$-analogs of Zagreb indices and coindices of graphs [19]. For further studies of Zagreb indices, see [20].

There are various operations on graphs such as union, intersection, complement, product, and subdivision. These operations on graphs are useful to obtain the new graphs from the old ones. Yan et al. listed five new graphs $L(G)$, $S(G), Q(G), R(G)$, and $T(G)$ with the help of five operations $L, S, Q, R$, and $T$ on a graph $G$, respectively, and studied the behavior of Wiener index of these graphs (see [4]). Eliasi and Taeri computed the Wiener indices of the $F$-sum graphs obtained by the Cartesian product of $F\left(G_{1}\right)$ and $G_{2}$, where $F \in\{S, R, Q, T\}$ [21]. Later on, many researchers worked on these $F$-sum graphs such as Deng et al. [22] computed first and second Zagreb indices, Akhtar and Imran calculated the forgotten index [23], Liu et al. computed first general Zagreb index [24], Ahmad et al. calculated sharp bounds of general sum-connectivity index [11], and Alanazi et al. calculated Gutman indices [25].

In this paper, we compute the bounds for the second Zagreb coindex of F-sum graphs in the form of Zagreb indices and coindices of their factor graphs. At the end, the obtained results are additionally illustrated with the assistance of examples of the exact and bonded values for some specific F-sum graphs. The rest of the paper is settled as follows: Section 2 contains the basic definitions and notions, Section 3 covers the main results, and Section 4 presents conclusion with specific examples related to the derived results.

\section{Preliminaries}

A graph denoted by $G=(V(G), E(G))$ is formed by set of vertices $V(G)$ and edges $E(G)$, where edge set is subset of the Cartesian product of set of vertices, i.e., $E(G) \subseteq V(G) \times V(G)$. In a simple connected graph $G=(V(G), E(G))$, total number of vertices is called its order (denoted by $|V(G)|$ ) and total number of edges is called its size (presented by $|E(G)|$ ). The degree of a vertex $u \in V(G)$ is number of its neighborhood vertices that is denoted by $d(u)$. The complement of $G$ is denoted by $\bar{G}$ and defined as $V(\bar{G})=V(G)$, and any two vertices (say $u$ and $v$ ) imply that $u v \in \bar{G}$ iff $u v \notin G$. Gutman and Trinajsti in 1972 [8] introduced the first and second Zagreb indices (denoted by $M_{1}$ and $M_{2}$ ) as follows:

$$
\begin{aligned}
& M_{1}(G)=\sum_{p_{1} p_{2} \in E(G)}\left[d_{G}\left(p_{1}\right)+d_{G}\left(p_{2}\right)\right], \\
& M_{2}(G)=\sum_{p_{1} p_{2} \in E(G)}\left[d_{G}\left(p_{1}\right) d_{G}\left(p_{2}\right)\right] .
\end{aligned}
$$

The second Zagreb coindex $\bar{M}_{2}(G)$ is defined in [13] as follows:

$$
\bar{M}_{2}(G)=\sum_{p_{1} p_{2} \notin E(G)}\left[d_{G}\left(p_{1}\right) d_{G}\left(p_{2}\right)\right] .
$$

It is important to note that the above defined coindex uses degrees of $G$ but run over $E(\bar{G})$.

Let $G$ be a graph, then

(i) $S(G)$ is a graph obtained by inserting one vertex in every edge of $G$

(ii) $R(G)$ is a graph obtained from $S(G)$ by joining the adjacent vertices of $G$

(iii) $Q(G)$ is a graph formed from $S(G)$ by joining the pairs of new vertices which are on the adjacent edges (the edges with one common vertex) of $G$

(iv) $T(G)$ is obtained by performing both operations of $R(G)$ and $Q(G)$ on $S(G)$, respectively

Let $G_{1}$ and $G_{2}$ be two simple connected graphs, then their $F$-sum graphs are denoted by $G_{1+F} G_{2}$ having vertex set $\left|V\left(G_{1+F} G_{2}\right)\right|=V\left(G_{1}\right) \cup E\left(G_{1}\right) \times V\left(G_{2}\right)$ and $\left(u_{1}, u_{2}\right)\left(v_{1}, v_{2}\right)$ $\in E\left(G_{1+F} G_{2}\right)$ iff

(i) $u_{1}=v_{1} \in V\left(G_{1}\right)$ and $u_{2} \backsim v_{2} \in G_{2}$

(ii) $u_{2}=v_{2} \in V\left(G_{2}\right) \quad$ and $\quad u_{1} \backsim v_{1} \in F\left(G_{1}\right)$, where $F \in\{S, R, Q, T\}$

For details, see Figures 1-3.

\section{Main Results}

In this section, main results of the second Zagreb coindex for the F-sum graphs are discussed.

Theorem 1. Let $G_{1}$ and $G_{2}$ be two simple connected graphs, then second Zagreb coindex of $G_{1+S} G_{2}$ is given as follows:

$$
\alpha_{1} \leq \bar{M}_{2}\left(G_{1+S} G_{2}\right) \leq \alpha_{2},
$$

where

$$
\begin{aligned}
\alpha_{1}= & 2 n_{2} e_{1}^{2}\left(\left(n_{1}-2\right)+n_{1}\left(n_{2}-1\right)\right)+2\left(n_{2}^{2} e_{1}^{2}-n_{2} e_{1}\right)+4 e_{2} e_{1}\left[\left(n_{1}-2\right)+n_{1}\left(n_{2}-1\right)\right]+2\left(e_{2}+\bar{e}_{2}\right) M_{1}\left(G_{1}\right)+2 e_{2} \bar{M}_{1}\left(G_{1}\right) \\
& +\left(n_{2}+2\left(e_{2}+\overline{e_{2}}\right)\right) M_{2}\left(G_{1}\right) \bar{M}_{2}\left(G_{1}\right)+\left(e_{1}+\overline{e_{1}}\right) M_{1}\left(G_{2}\right)+2 e_{1} \bar{M}_{1}\left(G_{2}\right)+2\left(e_{1}+\overline{e_{1}}\right) M_{2}\left(G_{2}\right) \\
& +\left(n_{1}+2\left(e_{1}+\overline{e_{1}}\right)\right) \bar{M}_{2}\left(G_{2}\right)+\left(M_{1}\left(G_{2}\right)+\bar{M}_{1}\left(G_{2}\right)\right)\left(M_{1}\left(G_{1}\right)+\bar{M}_{1}\left(G_{1}\right)\right), \\
\alpha_{2}= & 4 n_{2} e_{1} E\left(S\left(G_{1}\right)\right)\left(n_{2}-1+n_{2}\left(n_{1}-2\right)\right)+2\left(n_{2}^{2} e_{1}^{2}-n_{2} e_{1}\right)+4 e_{2} e_{1}\left[\left(n_{1}-2\right)+n_{1}\left(n_{2}-1\right)\right]+2\left(e_{2}+\overline{e_{2}}\right) M_{1}\left(G_{1}\right) \\
& +2 e_{2} \bar{M}_{1}\left(G_{1}\right)+\left(n_{2}+2\left(e_{2}+\bar{e}_{2}\right)\right) M_{2}\left(G_{1}\right) \bar{M}_{2}\left(G_{1}\right)+\left(e_{1}+\bar{e}_{1}\right) M_{1}\left(G_{2}\right)+2 e_{1} \bar{M}_{1}\left(G_{2}\right)+2\left(e_{1}+\bar{e}_{1}\right) M_{2}\left(G_{2}\right) \\
& +\left(n_{1}+2\left(e_{1}+\overline{e_{1}}\right)\right) \bar{M}_{2}\left(G_{2}\right)+\left(M_{1}\left(G_{2}\right)+\bar{M}_{1}\left(G_{2}\right)\right)\left(M_{1}\left(G_{1}\right)+\bar{M}_{1}\left(G_{1}\right)\right) .
\end{aligned}
$$




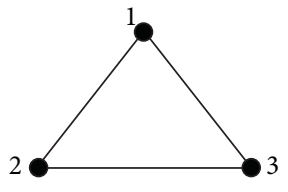

(a)

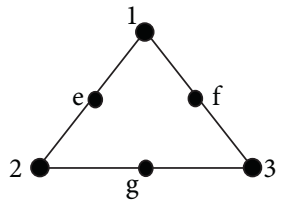

(b)

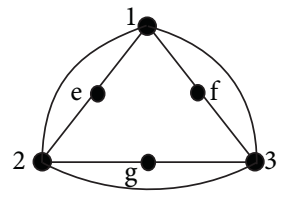

(c)

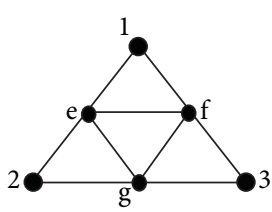

(d)

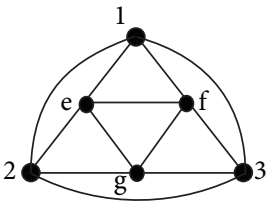

(e)

Figure 1: (a) $G \cong C_{3}$; (b) $S(G) \cong S\left(C_{3}\right)$; (c) $Q(G) \cong Q\left(C_{3}\right) ;\left(\right.$ d) $R(G) \cong R\left(C_{3}\right) ;\left(\right.$ e) $T(G) \cong T\left(C_{3}\right)$.

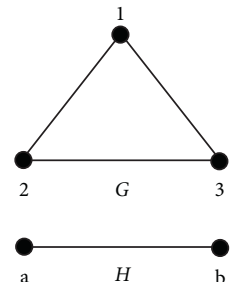

(a)

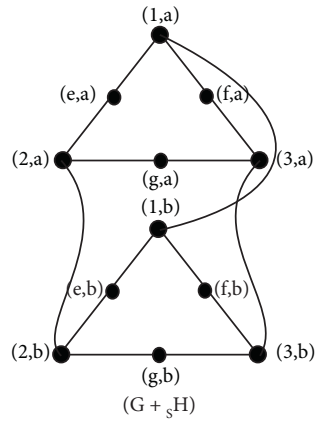

(b)

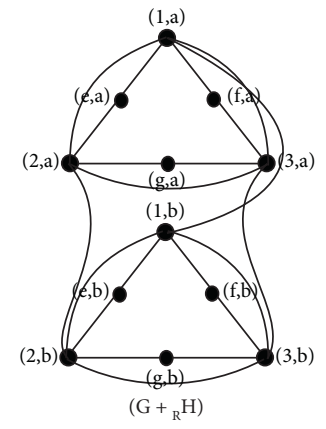

(c)

Figure $2: G \cong C_{3} ; H \cong P_{2} ; C_{3+S} P_{2} ; C_{3+R} P_{2}$.

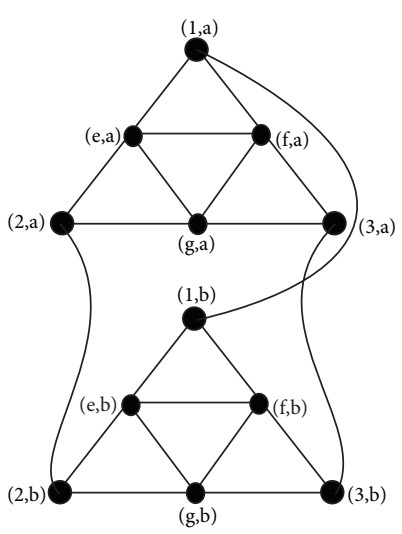

$\left(\mathrm{G}+{ }_{\mathrm{Q}} \mathrm{H}\right)$

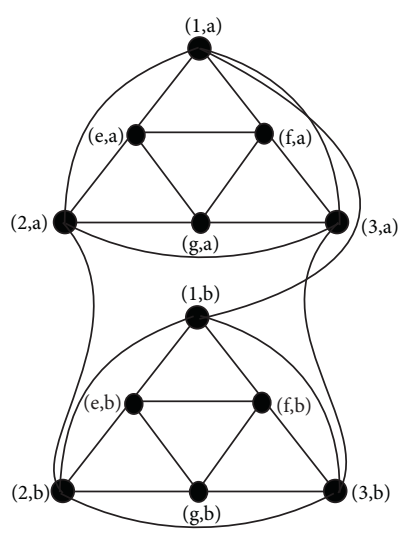

$\left(\mathrm{G}+{ }_{\mathrm{T}} \mathrm{H}\right)$

Figure 3: $C_{3+Q} P_{2}$ and $C_{3+T} P_{2}$.

Proof. Using equation (2), we have

$$
\begin{aligned}
\bar{M}_{2}\left(G_{1+S} G_{2}\right) & =\sum_{\left(p_{1}, p_{2}\right)}\left[d\left(q_{1}, q_{2}\right) \notin E\left(G_{1+S} G_{2}\right)\right. \\
\bar{M}_{2}\left(G_{1+S} G_{2}\right) & =\sum_{\left(p_{1}, p_{2}\right)}\left[\sum_{\left(q_{1}, q_{2}\right) \notin E\left(G_{1+S} G_{2}\right)}\left[d\left(p_{1}, q_{1}\right) d\left(p_{2}, q_{2}\right)\right]=\sum A+\sum B+\sum C,\right. \\
\sum A & =\sum_{p_{1}, p_{2} \in V\left(S\left(G_{1}\right)-V\left(G_{1}\right)\right)} \sum_{q_{1}, q_{2} \in V_{G_{2}}}\left[d\left(p_{1}, q_{1}\right)\left(p_{2}, q_{2}\right)\right] \\
& =\sum_{p_{1}, p_{2} \in V\left(S\left(G_{1}\right)-V\left(G_{1}\right)\right)} \sum_{q_{1}, q_{2} \in V_{G_{2}}}\left[d_{S\left(G_{1}\right)}\left(p_{1}\right) d_{S\left(G_{1}\right)}\left(p_{2}\right)\right]=\sum_{p_{1}, p_{2} \in V\left(S\left(G_{1}\right)-V\left(G_{1}\right)\right)} \sum_{q_{1}, q_{2} \in V_{G_{2}}}(2 \times 2), \\
\sum A & =2\left(n_{2}^{2} e_{1}^{2}-n_{2} e_{1}\right),
\end{aligned}
$$




$$
\begin{aligned}
& \sum B=\sum B_{1}+\sum B_{2}+\sum B_{3}+\sum B_{4}+\sum B_{5}+\sum B_{6}, \\
& \sum B_{1}=\sum_{p \in V_{G_{1}}} \sum_{q_{1} q_{2} \notin E_{G_{2}}}\left[d\left(t, q_{1}\right) d\left(t, q_{2}\right)\right] \\
& =\sum_{p \in V_{G_{1}}} \sum_{q_{1} q_{2} \notin E_{G_{2}}}\left[\left(d_{G_{1}}(p)+d_{G_{2}}\left(q_{1}\right) d_{G_{1}}(p)+d_{G_{2}}\left(q_{2}\right)\right)\right] \\
& =\sum_{p \in V_{G_{1}}} \sum_{q_{1} q_{2} \notin E_{G_{2}}}\left[d_{G_{1}}(p) d_{G_{1}}(p)+d_{G_{1}}(p) d_{G_{2}}\left(q_{2}\right)+d_{G_{1}}(p) d_{G_{2}}\left(q_{1}\right)+d_{G_{2}}\left(q_{1}\right) d_{G_{2}}\left(q_{2}\right)\right] \\
& =M_{1}\left(G_{1}\right) \bar{e}_{2}+2 e_{1} \bar{M}_{1}\left(G_{2}\right)+n_{1} \bar{M}_{2}\left(G_{2}\right), \\
& \sum B_{2}=\sum_{p_{1}, p_{2} \in V_{G_{1}}} \sum_{q \in V_{G_{2}}}\left[d\left(p_{1}, q\right) d\left(p_{2}, q\right)\right] \\
& =\sum_{q \in V_{G_{2}}} \sum_{p_{1} p_{2} \in E_{G_{1}}}\left[d\left(p_{1}, q\right) d\left(p_{2}, q\right)\right]+\sum_{q \in V_{G_{2}}} \sum_{p_{1} p_{2} \notin E_{G_{1}}}\left[d\left(p_{1}, q\right) d\left(p_{2}, q\right)\right] \\
& =\sum_{q \in V_{G_{2}}} \sum_{p_{1} p_{2} \in E_{G_{1}}}\left[\left(d_{G_{1}}\left(p_{1}\right)+d_{G_{2}}(q)\right)\left(d_{G_{1}}\left(p_{2}\right)+d_{G_{2}}(q)\right)\right]+\sum_{q \in V_{G_{2}}} \sum_{p_{1} p_{2} \notin E_{G_{1}}}\left[\left(d_{G_{1}}\left(p_{1}\right)+d_{G_{2}}(q)\right)\left(d_{G_{1}}\left(p_{2}\right)+d_{G_{2}}(q)\right)\right] \\
& =\sum_{q \in V_{G_{2}}} \sum_{p_{1} p_{2} \in E_{G_{1}}}\left[d_{G_{1}}\left(p_{1}\right) d_{G_{1}}\left(t_{2}\right)+d_{G_{1}}\left(p_{1}\right) d_{G_{2}}(q)+d_{G_{1}}\left(p_{2}\right) d_{G_{2}}(q)+d_{G_{2}}(q)^{2}\right] \\
& +\sum_{q \in V_{G_{2}}} \sum_{p_{1} p_{2} \notin E_{G_{1}}}\left[d_{G_{1}}\left(p_{1}\right) d_{G_{1}}\left(p_{2}\right)+d_{G_{1}}\left(p_{1}\right) d_{G_{2}}(q)+d_{G_{1}}\left(p_{2}\right) d_{G_{2}}(q)+d_{G_{2}}(q)^{2}\right] \\
& =n_{2} M_{2}\left(G_{1}\right)+2 e_{2} M_{1}\left(G_{1}\right)+e_{1} M_{1}\left(G_{2}\right)+n_{2} \bar{M}_{2}\left(G_{1}\right)+2 e_{2} \bar{M}_{1}\left(G_{1}\right)+\bar{e}_{1} M_{1}\left(G_{2}\right) \text {, } \\
& \sum B_{3}=\sum_{p_{1} p_{2} \in E_{G_{1}}} \sum_{q_{1} q_{2} \in E_{G_{2}}}\left[d\left(p_{1}, q_{1}\right) d\left(p_{2}, q_{2}\right)\right] \\
& =\sum_{p_{1} p_{2} \in E_{G_{1}}} \sum_{q_{1} q_{2} \in E_{G_{2}}}\left[\left(d_{G_{1}}\left(p_{1}\right)+d_{G_{2}}\left(q_{1}\right)\right)\left(d_{G_{1}}\left(p_{2}\right)+d_{G_{2}}\left(q_{2}\right)\right)\right] \\
& =\sum_{p_{1} p_{2} \in E_{G_{1}}} \sum_{q_{1} q_{2} \in E_{G_{2}}}\left[d_{G_{1}}\left(p_{1}\right) d_{G_{1}}\left(p_{2}\right)+d_{G_{1}}\left(p_{1}\right) d_{G_{2}}\left(q_{2}\right)+d_{G_{1}}\left(p_{2}\right) d_{G_{2}}\left(q_{1}\right)+d_{G_{2}}\left(q_{1}\right) d_{G_{2}}\left(q_{2}\right)\right] \\
& =2 e_{2} M_{2}\left(G_{1}\right)+M_{1}\left(G_{1}\right) M_{1}\left(G_{2}\right)+2 e_{1} M_{2}\left(G_{2}\right), \\
& \sum B_{4}=\sum_{p_{1} p_{2} \notin E_{G_{1}}} \sum_{q_{1} q_{2} \in E_{G_{2}}}\left[d\left(p_{1}, q_{1}\right) d\left(p_{2}, q_{2}\right)\right] \\
& =\sum_{p_{1} p_{2} \notin E_{G_{1}}} \sum_{q_{1} q_{2} \in E_{G_{2}}}\left[\left(d_{G_{1}}\left(p_{1}\right)+d_{G_{2}}\left(q_{1}\right)\right)\left(d_{G_{1}}\left(p_{2}\right)+d_{G_{2}}\left(q_{2}\right)\right)\right] \\
& =\sum_{p_{1} p_{2} \notin E_{G_{1}}} \sum_{q_{1} q_{2} \in E_{G_{2}}}\left[d_{G_{1}}\left(p_{1}\right) d_{G_{1}}\left(p_{2}\right)+d_{G_{1}}\left(p_{1}\right) d_{G_{2}}\left(q_{2}\right)+d_{G_{1}}\left(p_{2}\right) d_{G_{2}}\left(q_{1}\right)+d_{G_{2}}\left(q_{1}\right) d_{G_{2}}\left(q_{2}\right)\right] \\
& =2 e_{2} \bar{M}_{2}\left(G_{1}\right)+\bar{M}_{1}\left(G_{1}\right) M_{1}\left(G_{2}\right)+2 \bar{e}_{1} M_{2}\left(G_{2}\right), \\
& \sum B_{5}=\sum_{p_{1} p_{2} \notin E_{G_{1}}} \sum_{q_{1} q_{2} \notin E_{G_{2}}}\left[d\left(p_{1}, q_{1}\right) d\left(p_{2}, q_{2}\right)\right] \\
& =\sum_{p_{1} p_{2} \notin E_{G_{1}}} \sum_{q_{1} q_{2} \notin E_{G_{2}}}\left[\left(d_{G_{1}}\left(p_{1}\right)+d_{G_{2}}\left(q_{1}\right)\right)\left(d_{G_{1}}\left(p_{2}\right)+d_{G_{2}}\left(q_{2}\right)\right)\right] \\
& =\sum_{p_{1} p_{2} \notin E_{G_{1}}} \sum_{q_{1} q_{2} \notin E_{G_{2}}}\left[d_{G_{1}}\left(p_{1}\right) d_{G_{1}}\left(p_{2}\right)+d_{G_{1}}\left(p_{1}\right) d_{G_{2}}\left(q_{2}\right)+d_{G_{1}}\left(p_{2}\right) d_{G_{2}}\left(q_{1}\right)+d_{G_{2}}\left(q_{1}\right) d_{G_{2}}\left(q_{2}\right)\right] \\
& =2 \bar{e}_{2} \bar{M}_{2}\left(G_{1}\right)+\bar{M}_{1}\left(G_{1}\right) \bar{M}_{1}\left(G_{2}\right)+2 \bar{e}_{1} \bar{M}_{2}\left(G_{2}\right) \text {, } \\
& \sum B_{6}=\sum_{p_{1} p_{2} \in E_{G_{1}}} \sum_{q_{1} q_{2} \notin E_{G_{2}}}\left[d\left(p_{1}, q_{1}\right) d\left(p_{2}, q_{2}\right)\right] \\
& =\sum_{p_{1} p_{2} \in E_{G_{1}}} \sum_{q_{1} q_{2} \notin E_{G_{2}}}\left[\left(d_{G_{1}}\left(p_{1}\right)+d_{G_{2}}\left(q_{1}\right)\right)\left(d_{G_{1}}\left(p_{2}\right)+d_{G_{2}}\left(q_{2}\right)\right)\right] \\
& =\sum_{p_{1} p_{2} \in E_{G_{1}}} \sum_{q_{1} q_{2} \notin E_{G_{2}}}\left[d_{G_{1}}\left(p_{1}\right) d_{G_{1}}\left(p_{2}\right)+d_{G_{1}}\left(p_{1}\right) d_{G_{2}}\left(q_{2}\right)+d_{G_{1}}\left(p_{2}\right) d_{G_{2}}\left(q_{1}\right)+d_{G_{2}}\left(q_{1}\right) d_{G_{2}}\left(q_{2}\right)\right]
\end{aligned}
$$




$$
\begin{aligned}
& =2 \bar{e}_{2} M_{2}\left(G_{1}\right)+M_{1}\left(G_{1}\right) \bar{M}_{1}\left(G_{2}\right)+2 e_{1} \bar{M}_{2}\left(G_{2}\right), \\
& \sum B=2\left[\left(e_{2}+\overline{e_{2}}\right) M_{1}\left(G_{1}\right)+e_{2} \bar{M}_{1}\left(G_{1}\right)\right]+\left(n_{2}+2\left(e_{2}+\overline{e_{2}}\right)\right) M_{2}\left(G_{1}\right) \bar{M}_{2}\left(G_{1}\right)+\left(e_{1}+\overline{e_{1}}\right) M_{1}\left(G_{2}\right) \\
& +2\left[e_{1} \bar{M}_{1}\left(G_{2}\right)+\left(e_{1}+\overline{e_{1}}\right) M_{2}\left(G_{2}\right)\right]+\left(n_{1}+2\left(e_{1}+\bar{e}_{1}\right)\right) \bar{M}_{2}\left(G_{2}\right)+\left(M_{1}\left(G_{2}\right)+\bar{M}_{1}\left(G_{2}\right)\right)\left(M_{1}\left(G_{1}\right)+\bar{M}_{1}\left(G_{1}\right)\right) \text {, } \\
& \sum C=\sum C_{1}+\sum C_{2}+\sum C_{3} \\
& \sum C_{1}=\sum_{p_{1} p_{2} \notin E} \sum_{\left(S\left(G_{1}\right)\right)}\left[d\left(p_{1}, q\right) d\left(p_{2}, q\right)\right]=\sum_{p_{1} p_{2} \notin E\left(S\left(G_{G_{2}}\right)\right)} \sum_{q \in V_{G_{2}}}\left[\left(d_{G_{1}}\left(p_{1}\right)+d_{G_{2}}(q)\right)\left(d_{G_{1}}\left(p_{2}\right)\right)\right] \\
& p_{1} \in V\left(G_{1}\right) \\
& p_{2} \in V\left(S\left(G_{1}\right)-V\left(G_{1}\right)\right) \quad p_{2} \in V\left(S\left(G_{1}\right)-V\left(G_{1}\right)\right) \\
& =\sum_{p_{1} p_{2} \notin E\left(S\left(G_{1}\right)\right)} \sum_{q \in V_{G_{2}}}\left[\left(d_{G_{1}}\left(p_{1}\right)+d_{G_{2}}(q)\right) 2\right]+\sum_{p_{1} p_{2} \notin E\left(S\left(G_{1}\right)\right)} \sum_{q \in V_{G_{2}}}\left[2\left(d_{G_{1}}\left(p_{1}\right)+2 d_{G_{2}}(q)\right)\right] \\
& p_{1} \in V\left(G_{1}\right) \\
& p_{2} \in V\left(S\left(G_{1}\right)-V\left(G_{1}\right)\right) \\
& p_{1} \in V\left(G_{1}\right) \\
& p_{2} \in V\left(S\left(G_{1}\right)-V\left(G_{1}\right)\right) \\
& =2 n_{2} \sum_{p_{1} p_{2} \notin E\left(S\left(G_{1}\right)\right)} d\left(p_{1}\right)+4 e_{2} e_{1}\left(n_{1}-2\right) . \\
& p_{1} \in V\left(G_{1}\right) \\
& p_{2} \in V\left(S\left(G_{1}\right)-V\left(G_{1}\right)\right)
\end{aligned}
$$

Note that

$$
\begin{aligned}
& \begin{aligned}
e_{1} \leq & \sum_{\substack{p_{1} p_{2} \notin E\left(S\left(G_{1}\right)\right) \\
p_{1} \in V\left(G_{1}\right)}}\left[d\left(p_{1}\right)\right] \leq 2 e_{1}\left(n_{1}-2\right) E\left(S\left(G_{1}\right)\right) \\
& p_{2} \in V\left(S\left(G_{1}\right)-V\left(G_{1}\right)\right)
\end{aligned} \\
& 2 n_{2} e_{1}+4 e_{2} e_{1}\left(n_{1}-2\right) \leq \sum C_{1} \leq 4 n_{2} e_{1}\left(n_{1}-2\right) E\left(S\left(G_{1}\right)\right)+4 e_{2} e_{1}\left(n_{1}-2\right) \text {, }
\end{aligned}
$$

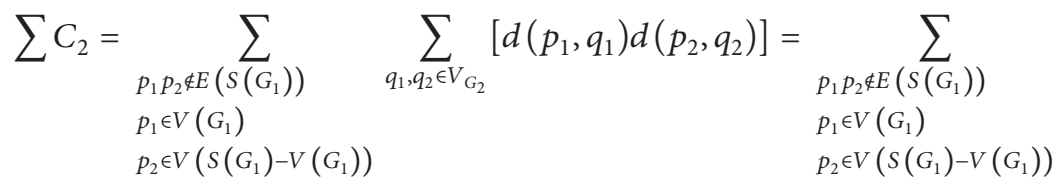

$$
\begin{aligned}
& \cdot \sum_{q_{1}, q_{2} \in V_{G_{2}}}\left[\left(d_{G_{1}}\left(p_{1}\right)+d\left(q_{1}\right)\right)\left(d_{S\left(G_{1}\right)}\left(p_{2}\right)\right)\right] \\
& \begin{aligned}
=\sum_{\substack{p_{1} p_{2} \notin E\left(S\left(G_{1}\right)\right) \\
p_{1} \in V\left(G_{1}\right)}} \sum_{q_{1}, q_{2} \in V_{G_{2}}}\left[\left(d_{G_{1}}\left(p_{1}\right)+d\left(q_{1}\right)\right) 2\right]= & \sum_{\substack{p_{1} p_{2} \notin E\left(S\left(G_{1}\right)\right) \\
p_{2} \in V\left(S\left(G_{1}\right)-V\left(G_{1}\right)\right)}} \sum_{p_{1} \in V\left(G_{1}\right)}\left[2 d_{q_{2} \in V_{G_{2}}}\left(p_{1}\right)+2 d\left(q_{1}\right)\right] \\
& p_{2} \in V\left(S\left(G_{1}\right)-V\left(G_{1}\right)\right)
\end{aligned} \\
& =2 n_{2}\left(n_{2}-1\right) \sum_{p_{1} p_{2} \notin E\left(S\left(G_{1}\right)\right)}\left[d_{G_{1}}\left(p_{1}\right)\right]+2\left(2 e_{2}\right) e_{1}\left(n_{1}-2\right)\left(n_{2}-1\right) \text {. } \\
& p_{1} \in V\left(G_{1}\right) \\
& p_{2} \in V\left(S\left(G_{1}\right)-V\left(G_{1}\right)\right)
\end{aligned}
$$


Note that

$$
\begin{aligned}
& e_{1} \leq \sum_{\substack{p_{1} p_{2} \notin E\left(S\left(G_{1}\right)\right) \\
p_{1} \in V\left(G_{1}\right) \\
\\
p_{2} \in V\left(S\left(G_{1}\right)-V\left(G_{1}\right)\right)}} d\left(p_{1}\right) \leq 2 e_{1}\left(n_{1}-2\right) E\left(S\left(G_{1}\right)\right), \\
& 2 n_{2}\left(n_{2}-1\right) e_{1}+4 e_{2} e_{1}\left(n_{1}-2\right)\left(n_{2}-1\right) \leq \sum C_{2} \leq 4 n_{2}\left(n_{2}-1\right) e_{1}\left(n_{1}-2\right) E\left(S\left(G_{1}\right)\right)+4 e_{2} e_{1}\left(n_{1}-2\right)\left(n_{2}-1\right), \\
& \sum C_{3}=\sum_{p_{1} p_{2} \notin E} \sum_{\left(S\left(G_{1}\right)\right)}\left[d\left(p_{1}, q_{1}\right)+d\left(p_{2}, q_{2}\right)\right] \\
& p_{1} \in V\left(G_{1}\right) \\
& p_{2} \in V\left(S\left(G_{1}\right)-V\left(G_{1}\right)\right) \\
& =\sum_{p_{1} p_{2} \notin E\left(S\left(G_{1}\right)\right)} \sum_{q_{1}, q_{2} \in V_{G_{2}}}\left[\left(d_{G_{1}}\left(p_{1}\right)+d\left(q_{1}\right)\right) d_{S\left(G_{1}\right)}\left(p_{2}\right)\right] \\
& p_{1} \in V\left(G_{1}\right) \\
& p_{2} \in V\left(S\left(G_{1}\right)-V\left(G_{1}\right)\right) \\
& =\sum_{p_{1} p_{2} \notin E\left(S\left(G_{1}\right)\right)} \sum_{q_{1}, q_{2} \in V_{G_{2}}}\left[\left(d_{G_{1}}\left(p_{1}\right)+d\left(q_{1}\right)\right) 2\right] \\
& p_{1} \in V\left(G_{1}\right) \\
& p_{2} \in V\left(S\left(G_{1}\right)-V\left(G_{1}\right)\right) \\
& =\sum_{\substack{p_{1} p_{2} \notin E\left(S\left(G_{1}\right)\right) \\
p_{1} \in V\left(G_{1}\right) \\
p_{2} \in V\left(S\left(G_{1}\right)-V\left(G_{1}\right)\right)}} \sum_{q_{1}, q_{2} \in V_{G_{2}}}\left[2 d_{G_{1}}\left(p_{1}\right)+2 d\left(q_{1}\right)\right] \\
& =2 n_{2}\left(n_{2}-1\right) \sum_{\substack{p_{1} p_{2} \notin E\left(S\left(G_{1}\right)\right) \\
p_{1} \in V\left(G_{1}\right) \\
p_{2} \in V\left(S\left(G_{1}\right)-V\left(G_{1}\right)\right)}}\left[d\left(p_{1}\right)\right]+2\left(2 e_{2}\right)\left(n_{2}-1\right) 2 e_{1} .
\end{aligned}
$$

Note that

$$
\begin{aligned}
2 e_{1} \leq & \sum_{\substack{p_{1} p_{2} \notin E\left(S\left(G_{1}\right)\right) \\
p_{1} \in V\left(G_{1}\right) \\
p_{2} \in V\left(S\left(G_{1}\right)-V\left(G_{1}\right)\right)}} d\left(p_{1}\right) \leq 2 e_{1} E\left(S\left(G_{1}\right)\right), \\
4 e_{1} n_{2}\left(n_{2}-1\right)+8 e_{1} e_{2}\left(n_{2}-1\right) \leq & \sum C_{3} \leq 4 e_{1} n_{2}\left(n_{2}-1\right) E\left(S\left(G_{1}\right)\right)+8 e_{1} e_{2}\left(n_{2}-1\right) .
\end{aligned}
$$

Consequently,

$$
\begin{aligned}
& 2 n_{2} e_{1}+4 e_{2} e_{1}\left(n_{1}-2\right)+2 n_{2}\left(n_{2}-1\right) e_{1}+4 e_{2} e_{1}\left(n_{1}-2\right)\left(n_{2}-1\right)+4 e_{1} n_{2}\left(n_{2}-1\right)+8 e_{1} e_{2}\left(n_{2}-1\right) \\
& \leq \sum C \leq 4 n_{2} e_{1}\left(n_{1}-2\right) E\left(S\left(G_{1}\right)\right)+4 e_{2} e_{1}\left(n_{1}-2\right)+4 n_{2}\left(n_{2}-1\right) e_{1}\left(n_{1}-2\right) E\left(S\left(G_{1}\right)\right) \\
& \quad+4 e_{2} e_{1}\left(n_{1}-2\right)\left(n_{2}-1\right)+4 e_{1} n_{2}\left(n_{2}-1\right) E\left(S\left(G_{1}\right)\right)+8 e_{1} e_{2}\left(n_{2}-1\right) .
\end{aligned}
$$

We obtained the required result by putting the values of $\sum A+\sum B+\sum C$ in equation (5).
Theorem 2. Let $G_{1}$ and $G_{2}$ be two simple connected graphs, then second Zagreb coindex of $G_{1+R} G_{2}$ is given as follows: 


$$
\alpha_{1} \leq \bar{M}_{2}\left(G_{1+R} G_{2}\right) \leq \alpha_{2},
$$

where

$$
\begin{aligned}
\alpha_{1}= & 4 n_{2} e_{1}\left(3 n_{2}-2\right)+2\left(n_{2}^{2} e_{1}^{2}-n_{2} e_{1}\right)+4 e_{2} e_{1}\left[n_{2}\left(n_{1}-2\right)+2\left(n_{2}-1\right)\right]+4 \overline{e_{2}} M_{1}\left(G_{1}\right)+4 e_{2} \bar{M}_{1}\left(G_{1}\right)+ \\
& 8\left(e_{2}+\overline{e_{2}}\right) M_{2}\left(G_{1}\right)+4 n_{2}+2\left(e_{2}+\bar{e}_{2}\right) \bar{M}_{2}\left(G_{1}\right)+\overline{e_{1}} M_{1}\left(G_{2}\right)+4 e_{1} \bar{M}_{1}\left(G_{2}\right)+2\left(e_{1}+\overline{e_{1}}\right) M_{2}\left(G_{2}\right) \\
& +\left(n_{1}+2\left(e_{1}+\overline{e_{1}}\right)\right) \bar{M}_{2}\left(G_{2}\right)+2\left(M_{1}\left(G_{2}\right)+\bar{M}_{1}\left(G_{2}\right)\right)\left(M_{1}\left(G_{1}\right)+\bar{M}_{1}\left(G_{1}\right)\right), \\
\alpha_{2}= & 8 n_{2} e_{1} E\left(R\left(G_{1}\right)\right)\left(n_{1}-2+\left(n_{2}-1\right)\left(n_{1}-1\right)\right)+2\left(n_{2}^{2} e_{1}^{2}-n_{2} e_{1}\right)+4 e_{2} e_{1}\left[n_{2}\left(n_{1}-2\right)+2\left(n_{2}-1\right)\right] \\
& +4 \overline{e_{2}} M_{1}\left(G_{1}\right)+4 e_{2} \bar{M}_{1}\left(G_{1}\right)+8\left(e_{2}+\overline{e_{2}}\right) M_{2}\left(G_{1}\right)+4 n_{2}+2\left(e_{2}+\bar{e}_{2}\right) \bar{M}_{2}\left(G_{1}\right)+\bar{e}_{1} M_{1}\left(G_{2}\right)+4 e_{1} \bar{M}_{1}\left(G_{2}\right) \\
& +2\left(e_{1}+\overline{e_{1}}\right) M_{2}\left(G_{2}\right)+\left(n_{1}+2\left(e_{1}+\overline{e_{1}}\right)\right) \bar{M}_{2}\left(G_{2}\right)+2\left(M_{1}\left(G_{2}\right)+\bar{M}_{1}\left(G_{2}\right)\right)\left(M_{1}\left(G_{1}\right)+\bar{M}_{1}\left(G_{1}\right)\right) .
\end{aligned}
$$

Proof. Using equation (2), we have

Using equation (6), we directly have

$$
\begin{aligned}
\bar{M}_{2}\left(G_{1+R} G_{2}\right)= & \sum_{\left(p_{1}, p_{2}\right)}\left[d\left(q_{1}, q_{2}\right) \notin E\left(G_{1+R}, q_{1}\right) d\left(p_{2}, q_{2}\right)\right] \\
& =\sum A+\sum B+\sum C .
\end{aligned}
$$

$$
\begin{aligned}
& \sum A=2\left(n_{2}^{2} e_{1}^{2}-n_{2} e_{1}\right) \\
& \sum B=\sum B_{1}+\sum B_{2}+\sum B_{3}+\sum B_{4}+\sum B_{5}+\sum B_{6} \\
& \sum B_{1}=\sum_{p \in V_{G_{1}}} \sum_{q_{1} q_{2} \notin E_{G_{2}}}\left[d\left(p, q_{1}\right) d\left(p, q_{2}\right)\right]=\sum_{p \in V_{G_{1}}} \sum_{q_{1} q_{2} \notin E_{G_{2}}}\left[\left(d_{R}(p)+d_{G_{2}}\left(q_{1}\right) d_{R}(p)+d_{G_{2}}\left(q_{2}\right)\right)\right] \\
& =\sum_{p \in V_{G_{1}}} \sum_{q_{1} q_{2} \notin E_{G_{2}}}\left[d_{R}(p) d_{R}(p)+d_{R}(p) d_{G_{2}}\left(q_{2}\right)+d_{R}(p) d_{G_{2}}\left(q_{1}\right)+d_{G_{2}}\left(q_{1}\right) d_{G_{2}}\left(q_{2}\right)\right] \\
& =\sum_{p \in V_{G_{1}}} \sum_{q_{1} q_{2} \notin E_{G_{2}}}\left[4 d_{G_{1}}(p)^{2}+2 d_{G_{1}}(p)\left(d_{G_{2}}\left(q_{2}\right)+d_{G_{2}}\left(q_{1}\right)\right)+d_{G_{2}}\left(q_{1}\right) d_{G_{2}}\left(q_{2}\right)\right] \\
& =4 \bar{e}_{2} M_{1}\left(G_{1}\right)+2\left(2 e_{1}\right) \bar{M}_{1}\left(G_{2}\right)+n_{1} \bar{M}_{2}\left(G_{2}\right), \\
& \sum B_{2}=\sum_{q \in V_{G_{2}}} \sum_{p_{1}, p_{2} \in V_{G_{1}}}\left[d\left(p_{1}, q\right) d\left(p_{2}, q\right)\right] \\
& =\sum_{q \in V_{G_{2}}} \sum_{p_{1} p_{2} \notin E_{G_{1}}}\left[d\left(p_{1}, q\right) d\left(p_{2}, q\right)\right]=\sum_{q \in V_{G_{2}}} \sum_{p_{1} p_{2} \notin E_{G_{1}}}\left[\left(d_{R}\left(p_{1}\right)+d_{G_{2}}(q)\right)\left(d_{R}\left(p_{2}\right)+d_{G_{2}}(q)\right)\right] \\
& =\sum_{q \in V_{G_{2}}} \sum_{p_{1} p_{2} \notin E_{G_{1}}}\left[\left(2 d_{G_{1}}\left(p_{1}\right)+d_{G_{2}}(q)\right)\left(2 d_{G_{1}}\left(p_{2}\right)+d_{G_{2}}(q)\right)\right] \\
& =\sum_{q \in V_{G_{2}}} \sum_{p_{1} p_{2} \notin E_{G_{1}}}\left[\left(4 d_{G_{1}}\left(p_{1}\right) d_{G_{1}}\left(p_{2}\right)+2 d_{G_{1}}\left(p_{1}\right) d_{G_{2}}(q)+2 d_{G_{1}}\left(p_{2}\right) d_{G_{2}}(q)+d_{G_{2}}(q)^{2}\right)\right] \\
& =4 n_{2} \bar{M}_{2}\left(G_{1}\right)+2\left(2 e_{2}\right) \bar{M}_{1}\left(G_{1}\right)+\bar{e}_{1} M_{1}\left(G_{2}\right) \text {, } \\
& \sum B_{3}=\sum_{p_{1} p_{2} \in E_{G_{1}}} \sum_{q_{1} q_{2} \in E_{G_{2}}}\left[d\left(p_{1}, q_{1}\right)+d\left(p_{2}, q_{2}\right)\right] \\
& =\sum_{p_{1} p_{2} \in E_{G_{1}}} \sum_{q_{1} q_{2} \in E_{G_{2}}}\left[\left(d_{R}(p) d_{R}(p)+d_{R}(p) d_{G_{2}}\left(q_{2}\right)+d_{R}(p) d_{G_{2}}\left(q_{1}\right)+d_{G_{2}}\left(q_{1}\right) d_{G_{2}}\left(q_{2}\right)\right)\right] \\
& =\sum_{p_{1} p_{2} \in E_{G_{1}}} \sum_{q_{1} q_{2} \in E_{G_{2}}}\left[\left(d_{R}(p) d_{R}(p)+d_{R}(p) d_{G_{2}}\left(q_{2}\right)+d_{R}(p) d_{G_{2}}\left(q_{1}\right)+d_{G_{2}}\left(q_{1}\right) d_{G_{2}}\left(q_{2}\right)\right)\right]
\end{aligned}
$$




$$
\begin{aligned}
& =2 \sum_{p_{1} p_{2} \in E_{G_{1}}} \sum_{q_{1} q_{2} \in E_{G_{2}}}\left[4 d_{G_{1}}(p)^{2}+2 d_{G_{1}}(p)\left(d_{G_{2}}\left(q_{2}\right)+d_{G_{2}}\left(q_{1}\right)\right)+d_{G_{2}}\left(q_{1}\right) d_{G_{2}}\left(q_{2}\right)\right] \\
& =2\left[4 e_{2} M_{2}\left(G_{1}\right)+e_{1} M_{2}\left(G_{2}\right)\right]+2 M_{1}\left(G_{1}\right) M_{1}\left(G_{2}\right), \\
& \sum B_{4}=\sum_{p_{1} p_{2} \notin E_{G_{1}}} \sum_{q_{1} q_{2} \in E_{G_{2}}}\left[d\left(p_{1}, q_{1}\right) d\left(p_{2}, q_{2}\right)\right] \\
& =\sum_{p_{1} p_{2} \notin E_{G_{1}}} \sum_{q_{1} q_{2} \in E_{G_{2}}}\left[\left(d_{R}\left(p_{1}\right)+d_{G_{2}}\left(q_{1}\right)\right)\left(d_{R}\left(p_{2}\right)+d_{G_{2}}\left(q_{2}\right)\right)\right] \\
& =\sum_{p_{1} p_{2} \notin E_{G_{1}}} \sum_{q_{1} q_{2} \in E_{G_{2}}}\left[\left(2 d_{G_{1}}\left(p_{1}\right)+d_{G_{2}}\left(q_{1}\right)\right)\left(2 d_{G_{1}}\left(p_{2}\right)+d_{G_{2}}\left(q_{2}\right)\right)\right] \\
& =\sum_{p_{1} p_{2} \notin E_{G_{1}}} \sum_{q_{1} q_{2} \in E_{G_{2}}}\left[4 d_{G_{1}}\left(p_{1}\right) d_{G_{1}}\left(p_{2}\right)+2\left[d_{G_{1}}\left(p_{1}\right) d_{G_{2}}\left(q_{2}\right)+d_{G_{1}}\left(p_{2}\right) d_{G_{2}}\left(q_{1}\right)\right]+d_{G_{2}}\left(q_{1}\right) d_{G_{2}}\left(q_{2}\right)\right] \\
& =2\left[4 e_{2} \bar{M}_{2}\left(G_{1}\right)+\bar{e}_{1} M_{2}\left(G_{2}\right)\right]+2 \bar{M}_{1}\left(G_{1}\right) M_{1}\left(G_{2}\right), \\
& \sum B_{5}=\sum_{p_{1} p_{2} \notin E_{G_{1}}} \sum_{q_{1} q_{2} \notin E_{G_{2}}}\left[d\left(p_{1}, q_{1}\right) d\left(p_{2}, q_{2}\right)\right] \\
& =\sum_{p_{1} p_{2} \notin E_{G_{1}}} \sum_{q_{1} q_{2} \notin E_{G_{2}}}\left[\left(d_{R}\left(p_{1}\right)+d_{G_{2}}\left(q_{1}\right)\right)\left(d_{R}\left(p_{2}\right)+d_{G_{2}}\left(q_{2}\right)\right)\right] \\
& =\sum_{p_{1} p_{2} \notin E_{G_{1}}} \sum_{q_{1} q_{2} \notin E_{G_{2}}}\left[\left(2 d_{G_{1}}\left(p_{1}\right)+d_{G_{2}}\left(q_{1}\right)\right)\left(2 d_{G_{1}}\left(p_{2}\right)+d_{G_{2}}\left(q_{2}\right)\right)\right] \\
& =\sum_{p_{1} p_{2} \notin E_{G_{1}}} \sum_{q_{1} q_{2} \notin E_{G_{2}}}\left[4 d_{G_{1}}\left(p_{1}\right) d_{G_{1}}\left(p_{2}\right)+2\left[d_{G_{1}}\left(p_{1}\right) d_{G_{2}}\left(q_{2}\right)+d_{G_{1}}\left(p_{2}\right) d_{G_{2}}\left(q_{1}\right)\right]+d_{G_{2}}\left(q_{1}\right) d_{G_{2}}\left(q_{2}\right)\right] \\
& =2\left[4 \bar{e}_{2} \bar{M}_{2}\left(G_{1}\right)+\bar{e}_{1} \bar{M}_{2}\left(G_{2}\right)\right]+2 \bar{M}_{1}\left(G_{1}\right) \bar{M}_{1}\left(G_{2}\right), \\
& \sum B_{6}=\sum_{p_{1} p_{2} \in E_{G_{1}}} \sum_{q_{1} q_{2} \notin E_{G_{2}}}\left[d\left(p_{1}, q_{1}\right) d\left(p_{2}, q_{2}\right)\right] \\
& =\sum_{p_{1} p_{2} \in E_{G_{1}}} \sum_{q_{1} q_{2} \notin E_{G_{2}}}\left[\left(d_{R}\left(p_{1}\right)+d_{G_{2}}\left(q_{1}\right)\right)\left(d_{R}\left(p_{2}\right)+d_{G_{2}}\left(q_{2}\right)\right)\right] \\
& =\sum_{p_{1} p_{2} \in E_{G_{1}}} \sum_{q_{1} q_{2} \in E_{G_{2}}}\left[\left(2 d_{G_{1}}\left(p_{1}\right)+d_{G_{2}}\left(q_{1}\right)\right)\left(2 d_{G_{1}}\left(p_{2}\right)+d_{G_{2}}\left(q_{2}\right)\right)\right] \\
& =\sum_{p_{1} p_{2} \in E_{G_{1}}} \sum_{q_{1} q_{2} \notin E_{G_{2}}}\left[4 d_{G_{1}}\left(p_{1}\right) d_{G_{1}}\left(p_{2}\right)+2\left[d_{G_{1}}\left(p_{1}\right) d_{G_{2}}\left(q_{2}\right)+d_{G_{1}}\left(p_{2}\right) d_{G_{2}}\left(q_{1}\right)\right]+d_{G_{2}}\left(q_{1}\right) d_{G_{2}}\left(q_{2}\right)\right] \\
& =2\left[4 \bar{e}_{2} M_{2}\left(G_{1}\right)+e_{1} \bar{M}_{2}\left(G_{2}\right)\right]+2 M_{1}\left(G_{1}\right) \bar{M}_{1}\left(G_{2}\right), \\
& \sum B=4 \overline{e_{2}} M_{1}\left(G_{1}\right)+4 e_{2} \bar{M}_{1}\left(G_{1}\right)+8\left(e_{2}+\overline{e_{2}}\right) M_{2}\left(G_{1}\right)+4 n_{2}+2\left(e_{2}+\overline{e_{2}}\right) \bar{M}_{2}\left(G_{1}\right)+\overline{e_{1}} M_{1}\left(G_{2}\right) \\
& +4 e_{1} \bar{M}_{1}\left(G_{2}\right)+2\left(e_{1}+\overline{e_{1}}\right) M_{2}\left(G_{2}\right)+\left(n_{1}++2\left(e_{1}+\overline{e_{1}}\right)\right) \\
& \bar{M}_{2}\left(G_{2}\right)+2\left(M_{1}\left(G_{2}\right)+\bar{M}_{1}\left(G_{2}\right)\right)\left(M_{1}\left(G_{1}\right)+\bar{M}_{1}\left(G_{1}\right)\right) \text {, } \\
& \sum C=\sum C_{1}+\sum C_{2}+\sum C_{3} \\
& \sum C_{1}=\sum_{\substack{p_{1} p_{2} \notin E \in\left(R\left(G_{1}\right)\right) \\
p_{1} \in\left(G_{1}\right)}} \sum_{q \in V_{G_{2}}} d p_{1}, q d p_{2}, q=\sum_{\substack{p_{1} p_{2} \notin E\left(R\left(G_{1}\right)\right) \\
p_{1} \in V\left(G_{1}\right)}} \sum_{q \in V_{G_{2}}}\left[\left(d_{R}\left(p_{1}\right)+d_{G_{2}}(q)\right)\left(d_{R}\left(p_{2}\right)\right)\right] \text {, } \\
& p_{2} \in V\left(R\left(G_{1}\right)-V\left(G_{1}\right)\right) \\
& \cdot \sum C_{1} \sum_{\substack{p_{1} p_{2} \notin E\left(R\left(G_{1}\right)\right) \\
p_{1} \in V\left(G_{1}\right) \\
p_{2} \in V\left(R\left(G_{1}\right)-V\left(G_{1}\right)\right)}} \sum_{q \in V_{G_{2}}}\left[\left(2 d_{G_{1}}\left(p_{1}\right)+d_{G_{2}}(q)\right) 2\right] \\
& =\sum_{\substack{p_{1} p_{2} \notin E\left(R\left(G_{1}\right)\right) \\
p_{1} \in V\left(G_{1}\right) \\
p_{2} \in V\left(s\left(G_{1}\right)-V\left(G_{1}\right)\right)}} \sum_{q \in V_{G_{2}}}\left[4\left(d_{G_{1}}\left(p_{1}\right)+2 d_{G_{2}}(q)\right)\right]=4 n_{2} \sum_{\substack{p_{1} p_{2} \notin E\left(R\left(G_{1}\right)\right) \\
p_{1} \in V\left(G_{1}\right) \\
p_{2} \in V\left(S\left(G_{1}\right)-V\left(G_{1}\right)\right)}}\left[d\left(p_{1}\right)\right]+4 e_{2} e_{1}\left(n_{1}-2\right) .
\end{aligned}
$$


Note that

$$
e_{1} \leq \sum_{\substack{p_{1} p_{2} \notin E\left(R\left(G_{1}\right)\right) \\ p_{1} \in V\left(G_{1}\right) \\ p_{2} \in V\left(S\left(G_{1}\right)-V\left(G_{1}\right)\right)}}\left[d\left(p_{1}\right)\right] \leq 2 e_{1}\left(n_{1}-2\right) E\left(S\left(G_{1}\right)\right) \text {, }
$$

so

$$
\begin{aligned}
& 4 n_{2} e_{1}+4 e_{2} e_{1}\left(n_{1}-2\right) \leq \sum C_{1} \leq 8 n_{2} e_{1}\left(n_{1}-2\right) E\left(R\left(G_{1}\right)\right)+4 e_{2} e_{1}\left(n_{1}-2\right), \\
& \sum C_{2}=\sum_{\substack{p_{1} p_{2} \notin E\left(R\left(G_{1}\right)\right) \\
p_{1} \in\left(G_{1}\right) \\
p_{2} \in V\left(R\left(G_{1}\right)-V\left(G_{1}\right)\right)}} \sum_{q_{1}, q_{2} \in V_{G_{2}}}\left[d\left(p_{1}, q_{1}\right) d\left(p_{2}, q_{2}\right)\right] \\
& =\sum_{\substack{p_{1} p_{2} \notin E\left(R\left(G_{1}\right)\right) \\
p_{1} \in V\left(G_{1}\right) \\
p_{2} \in V\left(R\left(G_{1}\right)-V\left(G_{1}\right)\right)}} \sum_{q_{1}, q_{2} \in V_{G_{2}}}\left[\left(d_{R}\left(p_{1}\right)+d\left(q_{1}\right)\right) d_{R\left(G_{1}\right)}\left(p_{2}\right)\right] \\
& =\sum_{p_{1} p_{2} \notin E\left(R\left(G_{1}\right)\right)} \sum_{q_{1}, q_{2} \in V_{G_{2}}}\left[\left(2 d_{G_{1}}\left(p_{1}\right)+d\left(q_{1}\right)\right) 2\right] \\
& p_{1} \in V\left(G_{1}\right) \\
& p_{2} \in V\left(R\left(G_{1}\right)-V\left(G_{1}\right)\right) \\
& =\sum_{p_{1} p_{2} \notin E\left(R\left(G_{1}\right)\right)} \sum_{q_{1}, q_{2} \in V_{G_{2}}}\left[4 d_{G_{1}}\left(p_{1}\right)+2 d\left(q_{1}\right)\right] \\
& p_{1} \in V\left(G_{1}\right) \\
& p_{2} \in V\left(R\left(G_{1}\right)-V\left(G_{1}\right)\right) \\
& =4 n_{2}\left(n_{2}-1\right) \sum_{p_{1} p_{2} \notin E\left(R\left(G_{1}\right)\right)}\left[d_{G_{1}}\left(p_{1}\right)\right]+2\left(2 e_{2}\right) e_{1}\left(n_{1}-2\right)\left(n_{2}-1\right) . \\
& p_{1} \in V\left(G_{1}\right) \\
& p_{2} \in V\left(R\left(G_{1}\right)-V\left(G_{1}\right)\right)
\end{aligned}
$$

Note that

$$
e_{1} \leq \sum_{\substack{p_{1} p_{2} \notin E\left(R\left(G_{1}\right)\right) \\ p_{1} \in V\left(G_{1}\right) \\ p_{2} \in V\left(R\left(G_{1}\right)-V\left(G_{1}\right)\right)}} d\left(p_{1}\right) \leq 2 e_{1}\left(n_{1}-2\right) E\left(R\left(G_{1}\right)\right),
$$


so

$$
\begin{aligned}
& 4 n_{2}\left(n_{2}-1\right) e_{1}+4 e_{2} e_{1}\left(n_{1}-2\right)\left(n_{2}-1\right) \leq \sum C_{2} \leq 8 n_{2}\left(n_{2}-1\right) e_{1}\left(n_{1}-2\right) E\left(R\left(G_{1}\right)\right)+4 e_{2} e_{1}\left(n_{1}-2\right)\left(n_{2}-1\right), \\
& \sum C_{3}=\sum_{p_{1} p_{2} \notin E\left(R\left(G_{1}\right)\right)} \sum_{q_{1}, q_{2} \in V_{G_{2}}}\left[d\left(p_{1}, q_{1}\right)\left(d\left(p_{2}, q_{2}\right)\right)\right] \\
& p_{1} \in V\left(G_{1}\right) \\
& p_{2} \in V\left(R\left(G_{1}\right)-V\left(G_{1}\right)\right) \\
& =\sum_{\substack{p_{1} p_{2} \notin E\left(R\left(G_{1}\right)\right) \\
p_{1} \in V\left(G_{1}\right) \\
p_{2} \in V\left(R\left(G_{1}\right)-V\left(G_{1}\right)\right)}} \sum_{q_{1}, q_{2} \in V_{G_{2}}}\left[\left(d_{R}\left(p_{1}\right)+d\left(q_{1}\right)\right)\left(d_{R\left(G_{1}\right)}\left(p_{2}\right)\right)\right]
\end{aligned}
$$

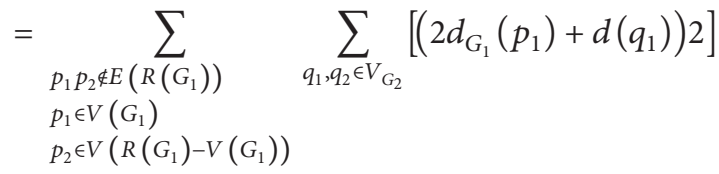

$$
\begin{aligned}
& =\sum_{\substack{p_{1} p_{2} \notin E\left(R\left(G_{1}\right)\right) \\
p_{1} \in V\left(G_{1}\right) \\
p_{2} \in V\left(R\left(G_{1}\right)-V\left(G_{1}\right)\right)}} \sum_{q_{1}, q_{2} \in V_{G_{2}}}\left[4 d_{G_{1}}\left(p_{1}\right)+2 d\left(q_{1}\right)\right] \\
& =4 n_{2}\left(n_{2}-1\right) \sum_{\substack{p_{1} p_{2} \notin E\left(R\left(G_{1}\right)\right) \\
p_{1} \in V\left(G_{1}\right) \\
p_{2} \in V\left(R\left(G_{1}\right)-V\left(G_{1}\right)\right)}}\left[d\left(p_{1}\right)\right]+2\left(2 e_{2}\right)\left(n_{2}-1\right) 2 e_{1} .
\end{aligned}
$$

Note that

$$
2 e_{1} \leq \sum_{\substack{p_{1} p_{2} \notin E\left(R\left(G_{1}\right)\right) \\ p_{1} \in V\left(G_{1}\right) \\ \\ p_{2} \in V\left(R\left(G_{1}\right)-V\left(G_{1}\right)\right)}} d\left(p_{1}\right) \leq 2 e_{1} E\left(R\left(G_{1}\right)\right),
$$

so

$$
\begin{aligned}
& 8 e_{1} n_{2}\left(n_{2}-1\right)+8 e_{1} e_{2}\left(n_{2}-1\right) \\
& \leq \sum C_{3} \leq 8 e_{1} n_{2}\left(n_{2}-1\right) E\left(R\left(G_{1}\right)\right)+8 e_{1} e_{2}\left(n_{2}-1\right) .
\end{aligned}
$$

$$
\begin{aligned}
& 8 e_{1} n_{2}\left(n_{2}-1\right)+8 e_{1} e_{2}\left(n_{2}-1\right)+4 n_{2}\left(n_{2}-1\right) e_{1}+4 e_{2} e_{1}\left(n_{1}-2\right)\left(n_{2}-1\right)+4 n_{2} e_{1}+4 e_{2} e_{1}\left(n_{1}-2\right) \\
& \leq \sum C \\
& \leq 8 n_{2} e_{1}\left(n_{1}-2\right) E\left(R\left(G_{1}\right)\right)+4 e_{2} e_{1}\left(n_{1}-2\right)+8 n_{2}\left(n_{2}-1\right) e_{1}\left(n_{1}-2\right) E\left(R\left(G_{1}\right)\right)+4 e_{2} e_{1}\left(n_{1}-2\right)\left(n_{2}-1\right) \\
& \quad+8 e_{1} n_{2}\left(n_{2}-1\right) E\left(R\left(G_{1}\right)\right)+8 e_{1} e_{2}\left(n_{2}-1\right) .
\end{aligned}
$$

We obtained the required proof by putting the values of $\sum A+\sum B+\sum C$ in equation (14).
Theorem 3. Let $G_{1}$ and $G_{2}$ be two simple connected graphs, then second Zagreb coindex of $G_{1+Q} G_{2}$ is given as follows: 


$$
\alpha_{1} \leq \bar{M}_{2}\left(G_{1+Q} G_{2}\right) \leq \alpha_{2},
$$

where

$$
\begin{aligned}
\alpha_{1}= & 4 e_{2}\left[\bar{e}_{1}+\left(n_{2}-1\right)\left(\bar{e}_{1}+e_{1}\right)\right]+n_{2}^{2} \bar{M}_{2}\left(G_{1}\right)+n_{2}\left(n_{2}-1\right) M_{2}\left(G_{1}\right)+\left(n_{2}-1+\bar{e}_{2}\right)\left(M_{1}\left(G_{1}\right)+2 M_{2}\left(G_{1}\right)\right) \\
& +\left(2 e_{2}+\bar{e}_{2}\right) M_{1}\left(G_{1}\right)+2 e_{2} \bar{M}_{1}\left(G_{1}\right)+\left(n_{2}+2\left(e_{2}+\overline{e_{2}}\right)\right) M_{2}\left(G_{1}\right) \bar{M}_{2}\left(G_{1}\right)+\left(e_{1}+\bar{e}_{1}\right) M_{1}\left(G_{2}\right)+2 e_{1} \bar{M}_{1}\left(G_{2}\right) \\
& +2\left(e_{1}+\overline{e_{1}}\right) M_{2}\left(G_{2}\right)+\left(n_{1}+2\left(e_{1}+\bar{e}_{1}\right)\right) \bar{M}_{2}\left(G_{2}\right)+\left(M_{1}\left(G_{2}\right)+\bar{M}_{1}\left(G_{2}\right)\right)\left(M_{1}\left(G_{1}\right)+\bar{M}_{1}\left(G_{1}\right)\right), \\
\alpha_{2}= & 4 e_{2}\left[\bar{e}_{Q}\left(G_{1}\right)+\left(n_{2}-1\right)\left(\bar{e}_{Q\left(G_{1}\right)}+e_{Q}\left(G_{1}\right)\right)\right]+n_{2}^{2} \bar{M}_{2}\left(Q\left(G_{1}\right)\right)+n_{2}\left(n_{2}-1\right) M_{2}\left(Q\left(G_{1}\right)\right) \\
& +\left(n_{2}+2\left(n_{2}-1+\bar{e}_{2}\right)\right) M_{2}\left(Q\left(G_{1}\right)\right)+\left(n_{2}-1+\bar{e}_{2}\right) M_{1}\left(Q\left(G_{1}\right)\right)+2\left(n_{2}-1+\bar{e}_{2}\right) M_{2}\left(Q\left(G_{1}\right)\right)+\left(2 e_{2}+\overline{e_{2}}\right) M_{1}\left(G_{1}\right) \\
& +2 e_{2} \bar{M}_{1}\left(G_{1}\right)+\left(n_{2}+2\left(e_{2}+\bar{e}_{2}\right)\right) M_{2}\left(G_{1}\right) \bar{M}_{2}\left(G_{1}\right)+\left(e_{1}+\bar{e}_{1}\right) M_{1}\left(G_{2}\right)+2 e_{1} \bar{M}_{1}\left(G_{2}\right)+2\left(e_{1}+\overline{e_{1}}\right) M_{2}\left(G_{2}\right) \\
& +\left(n_{1}+2\left(e_{1}+\bar{e}_{1}\right)\right) \bar{M}_{2}\left(G_{2}\right)+\left(M_{1}\left(G_{2}\right)+\bar{M}_{1}\left(G_{2}\right)\right)\left(M_{1}\left(G_{1}\right)+\bar{M}_{1}\left(G_{1}\right)\right) .
\end{aligned}
$$

Proof. Using equation (2), we have

$$
\begin{aligned}
\bar{M}_{2}\left(G_{1+Q} G_{2}\right)= & \sum_{\left(p_{1}, p_{2}\right)}\left[d\left(p_{1}, x_{2}\right) \notin E\left(G_{1+Q} G_{2}\right) d\left(p_{2}, x_{2}\right)\right]=\sum A+\sum B+\sum C, \\
\sum A= & \sum A_{1}+\sum A_{2}+\sum A_{3}+\sum A_{4}+\sum A_{5}+\sum A_{6}+\sum A_{7}, \\
\sum A_{1}= & \sum_{\substack{p_{1} p_{2} \notin E \\
p_{1}, p_{2} \in V\left(Q\left(G_{1}\right)\right)}} \sum \sum_{x \in V_{G_{2}}}\left[d p_{1}, x d p_{2}, x\right]=n_{2} \sum_{p_{1} p_{2} \notin E\left(Q\left(G_{1}\right)\right)}\left[d_{p_{1}, p_{2} \in V\left(Q\left(G_{1}\right)-\left(G_{1}\right)\right)} p_{Q G_{1}} p_{1} d_{Q G_{1}} p_{2}\right] .
\end{aligned}
$$

Note that

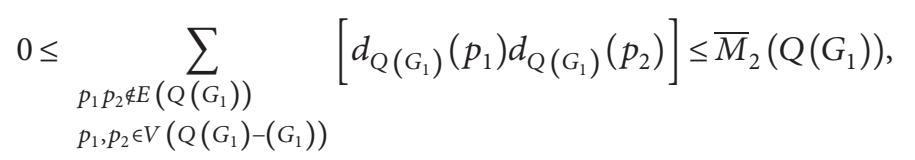

$$
\begin{aligned}
0 & \leq \sum A_{1} \leq n_{2} \bar{M}_{2}\left(Q\left(G_{1}\right)\right), \\
\sum A_{2} & =\sum_{p \in V\left(Q\left(G_{1}\right)-\left(G_{1}\right)\right)} \sum_{x_{1} x_{2} \in E_{G_{2}}}\left[d\left(p, x_{1}\right) d\left(p, x_{2}\right)\right] \\
& =\sum_{p \in V\left(Q\left(G_{1}\right)-\left(G_{1}\right)\right)} \sum_{x_{1} x_{2} \in E_{G_{2}}}\left[d_{Q\left(G_{1}\right)}(p) d_{Q\left(G_{1}\right)}(p)\right]=\left(n_{2}-1\right) \sum_{p \in V\left(Q\left(G_{1}\right)-\left(G_{1}\right)\right)}\left[d_{Q\left(G_{1}\right)}(p)^{2}\right] .
\end{aligned}
$$


Note that

$M_{1}\left(G_{1}\right) \leq \sum_{p \in V\left(Q\left(G_{1}\right)-\left(G_{1}\right)\right)}\left[d_{Q\left(G_{1}\right)}(p)^{2}\right] \leq M_{1}\left(Q\left(G_{1}\right)\right)$

(30)

so

$$
\begin{aligned}
\left(n_{2}-1\right) M_{1}\left(G_{1}\right) & \leq \sum A_{2} \leq\left(n_{2}-1\right) M_{1}\left(Q\left(G_{1}\right)\right) \\
\sum A_{3} & =\sum_{p \in V\left(Q\left(G_{1}\right)-\left(G_{1}\right)\right)} \sum_{x_{1} x_{2} \notin E_{G_{2}}}\left[d\left(p, x_{1}\right) d\left(p, x_{2}\right)\right] \\
& =\sum_{p \in V\left(Q\left(G_{1}\right)-V\left(G_{1}\right)\right)} \sum_{x_{1} x_{2} \notin E_{G_{2}}}\left[d_{Q\left(G_{1}\right)}(p) d_{Q\left(G_{1}\right)}(p)\right]=\bar{e}_{2} \sum_{t \in V\left(Q\left(G_{1}\right)-V\left(G_{1}\right)\right)}\left[d_{Q\left(G_{1}\right)}(p)^{2}\right] .
\end{aligned}
$$

Note that

$$
M_{1}\left(G_{1}\right) \leq \sum_{p \in V\left(Q\left(G_{1}\right)-V\left(G_{1}\right)\right)}\left[d_{Q\left(G_{1}\right)}(p)^{2}\right] \leq M_{1}\left(Q\left(G_{1}\right)\right)
$$

$$
\begin{aligned}
\bar{e}_{2} M_{1}\left(G_{1}\right) \leq & \sum A_{3} \leq \bar{e}_{2} M_{1}\left(Q\left(G_{1}\right)\right) \\
\sum A_{4}= & \sum_{\substack{p_{1} p_{2} \in E\left(Q\left(G_{1}\right)\right) \\
p_{1}, p_{2} \in V\left(Q\left(G_{1}\right)-V\left(G_{1}\right)\right)}} \sum_{\substack{1 \\
x_{2} \in E_{G_{2}}}}\left[d\left(p_{1}, x_{1}\right) d\left(p_{2}, x_{2}\right)\right] \\
= & \sum_{\substack{p_{1} p_{2} \in E\left(Q\left(G_{1}\right)\right) \\
p_{1}, p_{2} \in V\left(Q\left(G_{1}\right)-V\left(G_{1}\right)\right)}} \sum_{\substack{x_{1} x_{2} \in E_{G_{2}} \\
p_{1}}}\left[d p_{1}, x_{1} d p_{2}, x_{2}\right]=2 n_{2}-1 \sum_{\substack{p_{1} p_{2} \in E\left(Q\left(G_{1}\right)\right) \\
p_{1}, p_{2} \in V\left(Q\left(G_{1}\right)-V\left(G_{1}\right)\right)}}\left[d_{Q G_{1}} p_{1} d d_{Q G_{1}} p_{2}\right] .
\end{aligned}
$$

Note that

$$
M_{2}\left(G_{1}\right) \leq \sum_{\substack{p_{1} p_{2} \in E\left(Q\left(G_{1}\right)\right) \\ p_{1}, p_{2} \in V\left(Q\left(G_{1}\right)-V\left(G_{1}\right)\right)}}\left[d_{Q\left(G_{1}\right)}\left(p_{1}\right)+d_{Q\left(G_{1}\right)}\left(p_{2}\right)\right] \leq M_{2}\left(Q\left(G_{1}\right)\right)
$$


Mathematical Problems in Engineering

13

so

$$
\begin{aligned}
2\left(n_{2}-1\right) M_{2}\left(G_{1}\right) \leq & \sum A_{4} \leq 2\left(n_{2}-1\right) M_{2}\left(Q\left(G_{1}\right)\right) \\
\sum A_{5}= & \sum_{\substack{p_{1} p_{2} \in E\left(Q\left(G_{1}\right)\right) \\
p_{1}, p_{2} \in V\left(Q\left(G_{1}\right)-V\left(G_{1}\right)\right)}} \sum_{\substack{x_{1} x_{2} \in E_{G_{2}}\\
}}\left[d\left(p_{1}, x_{1}\right) d\left(p_{2}, x_{2}\right)\right] \\
& \sum_{\substack{p_{1} p_{2} \in E\left(Q\left(G_{1}\right)\right) \\
p_{1}, p_{2} \in V\left(Q\left(G_{1}\right)-V\left(G_{1}\right)\right)}} \sum_{\substack{x_{1} x_{2} \in E_{G_{2}} \\
p_{1}}}\left[d\left(p_{1}, x_{1}\right) d\left(p_{2}, x_{2}\right)\right]=2 \bar{e}_{2} \sum_{\substack{p_{1} p_{2} \in E\left(Q\left(G_{1}\right)\right) \\
p_{1}, p_{2} \in V\left(Q\left(G_{1}\right)-V\left(G_{1}\right)\right)}}\left[d_{Q\left(G_{1}\right)}\left(p_{1}\right) d_{Q\left(G_{1}\right)}\left(p_{2}\right)\right] .
\end{aligned}
$$

Note that

$$
M_{2}\left(G_{1}\right) \leq \sum_{p_{1} p_{2} \in E\left(Q\left(G_{1}\right)\right)} \quad\left[d_{Q\left(G_{1}\right)}\left(p_{1}\right)+d_{Q\left(G_{1}\right)}\left(p_{2}\right)\right] \leq M_{2}\left(Q\left(G_{1}\right)\right) \text {, }
$$

so

$$
\begin{aligned}
2 \bar{e}_{2} M_{2}\left(G_{1}\right) \leq & \sum A_{5} \leq 2 \bar{e}_{2} M_{2}\left(Q\left(G_{1}\right)\right), \\
\sum A_{6}= & \sum_{\substack{p_{1} p_{2} \in E\left(Q\left(G_{1}\right)\right) \\
p_{1}, p_{2} \in V\left(Q\left(G_{1}\right)-V\left(G_{1}\right)\right)}} \sum_{\substack{x_{1} x_{2} \in E_{G_{2}} \\
p_{1} p_{2} \in E\left(Q\left(G_{1}\right)\right) \\
p_{1}, p_{2} \in V\left(Q\left(G_{1}\right)-V\left(G_{1}\right)\right)}} \sum_{\substack{x_{1} x_{2} \in E_{G_{2}} \\
p_{1}}}\left[d\left(p_{1}, x_{1}\right) d\left(p_{2}, x_{2}\right)\right]=2\left(n_{2}-1\right) \sum_{\substack{p_{1} p_{2} \in E\left(Q\left(G_{1}\right)\right) \\
p_{1}, p_{2} \in V\left(Q\left(G_{1}\right)-V\left(G_{1}\right)\right)}}\left[d_{Q\left(G_{1}\right)}\left(p_{1}\right) d_{Q\left(G_{1}\right)}\left(p_{2}\right)\right] .
\end{aligned}
$$

Note that

$$
0 \leq \sum_{\substack{p_{1} p_{2} \in E\left(Q\left(G_{1}\right)\right) \\ p_{1}, p_{2} \in V\left(Q\left(G_{1}\right)-V\left(G_{1}\right)\right)}}\left[d_{Q\left(G_{1}\right)}\left(p_{1}\right) d_{Q\left(G_{1}\right)}\left(p_{2}\right)\right] \leq \bar{M}_{2}\left(Q\left(G_{1}\right)\right),
$$

so 
Note that

$0 \leq \sum_{\substack{p_{1} p_{2} \notin E\left(Q\left(G_{1}\right)\right) \\ p_{1}, p_{2} \in V\left(Q\left(G_{1}\right)-V\left(G_{1}\right)\right)}}\left[d_{Q\left(G_{1}\right)}\left(p_{1}\right) d_{Q\left(G_{1}\right)}\left(p_{2}\right)\right] \leq \bar{M}_{2}\left(Q\left(G_{1}\right)\right)$, so

$$
0 \leq \sum A_{7} \leq 2 \bar{e}_{2} \bar{M}_{2}\left(Q\left(G_{1}\right)\right)
$$

$$
\begin{aligned}
& 2 \bar{e}_{2} M_{2}\left(G_{1}\right)+2\left(n_{2}-1\right) M_{2}\left(G_{1}\right)+\left(n_{2}-1\right) M_{1}\left(G_{1}\right)+\bar{e}_{2} M_{1}\left(G_{1}\right) \\
& \leq \sum A \\
& \leq n_{2} \bar{M}_{2}\left(Q\left(G_{1}\right)\right)+\left(n_{2}-1\right) M_{1}\left(Q\left(G_{1}\right)\right)+\bar{e}_{2} M_{1}\left(Q\left(G_{1}\right)\right)+2\left(n_{2}-1\right) M_{2}\left(Q\left(G_{1}\right)\right)+2 \bar{e}_{2} M_{2}\left(Q\left(G_{1}\right)\right) \\
& \quad+2\left(n_{2}-1\right) \bar{M}_{2}\left(Q\left(G_{1}\right)\right)+2 \bar{e}_{2} \bar{M}_{2}\left(Q\left(G_{1}\right)\right) .
\end{aligned}
$$

Using equation (7), we directly have

$$
\begin{aligned}
& \sum B=2\left[\left(e_{2}+\overline{e_{2}}\right) M_{1}\left(G_{1}\right)+e_{2} \bar{M}_{1}\left(G_{1}\right)\right]+\left(n_{2}+2\left(e_{2}+\overline{e_{2}}\right)\right) M_{2}\left(G_{1}\right) \bar{M}_{2}\left(G_{1}\right)+\left(e_{1}+\overline{e_{1}}\right) M_{1}\left(G_{2}\right) \\
& +2\left[e_{1} \bar{M}_{1}\left(G_{2}\right)+\left(e_{1}+\overline{e_{1}}\right) M_{2}\left(G_{2}\right)\right]+\left(n_{1}+2\left(e_{1}+\overline{e_{1}}\right)\right) \bar{M}_{2}\left(G_{2}\right) \\
& +\left(M_{1}\left(G_{2}\right)+\bar{M}_{1}\left(G_{2}\right)\right)\left(M_{1}\left(G_{1}\right)+\bar{M}_{1}\left(G_{1}\right)\right), \\
& \sum C=\sum C_{1}+\sum C_{2}+\sum C_{3} \\
& \begin{aligned}
\sum C_{1}= & \sum_{\substack{p_{1} p_{2} \notin E\left(Q\left(G_{1}\right)\right) \\
p_{1} \in V\left(G_{1}\right)}} \sum_{x \in V_{G_{2}}} d p_{1}, x d p_{2}, x=\sum_{\substack{p_{1} p_{2} \notin E\left(Q\left(G_{1}\right)\right) \\
p_{1} \in V\left(G_{1}\right)}} \sum_{x \in V_{G_{2}}}\left[\left(d_{G_{1}}\left(p_{1}\right)+d(x)\right) d_{Q\left(G_{1}\right)}\left(p_{2}\right)\right]{ }_{2} \in V\left(Q\left(G_{1}\right)-V\left(G_{1}\right)\right)
\end{aligned} \\
& =n_{2} \sum_{p_{1} p_{2} \notin E\left(Q\left(G_{1}\right)\right)}\left[d_{G_{1}}\left(p_{1}\right) d_{Q\left(G_{1}\right)}\left(p_{2}\right)+2 e_{2} d_{Q\left(G_{1}\right)}\left(p_{2}\right)\right] \\
& p_{1} \in V\left(G_{1}\right) \\
& p_{2} \in V\left(Q\left(G_{1}\right)-V\left(G_{1}\right)\right) \\
& \begin{array}{cl}
=n_{2} \sum_{\substack{p_{1} p_{2} \notin E\left(Q\left(G_{1}\right)\right) \\
p_{1} \in V\left(G_{1}\right)}} d_{G_{1}}\left(p_{1}\right) d_{Q\left(G_{1}\right)}\left(p_{2}\right)+d(x) \sum_{\substack{p_{1} p_{2} \notin E\left(Q\left(G_{1}\right)\right) \\
p_{2} \in V\left(Q\left(G_{1}\right)-V\left(G_{1}\right)\right)}} d_{1} \in V\left(G_{1}\right) \\
p_{2} \in V\left(Q\left(G_{1}\right)-V\left(G_{1}\right)\right)
\end{array}
\end{aligned}
$$


Note that

$$
\begin{aligned}
& \begin{aligned}
\bar{M}_{2}\left(G_{1}\right) \leq & \sum_{\substack{p_{1} p_{2} \notin E\left(Q\left(G_{1}\right)\right) \\
p_{1} \in V\left(G_{1}\right)}} d_{Q\left(G_{1}\right)}\left(p_{1}\right) \leq \bar{M}_{2} Q\left(G_{1}\right) \\
& p_{2} \in V\left(Q\left(G_{1}\right)-V\left(G_{1}\right)\right)
\end{aligned} \\
& \begin{aligned}
2 \bar{e}_{1} \leq & \sum_{\substack{p_{1} p_{2} \notin E\left(Q\left(G_{1}\right)\right)\\
}} d_{1} \in V\left(G_{1}\right) \\
& p_{2} \in V\left(Q\left(G_{1}\right)-V\left(G_{1}\right)\right)
\end{aligned} \\
& n_{2} \bar{M}_{2}\left(G_{1}\right)+4 e_{2} \bar{e}_{1} \leq \sum C_{1} \leq n_{2} \bar{M}_{2}\left(Q\left(G_{1}\right)\right)+4 e_{2} \bar{e}_{Q\left(G_{1}\right)} \text {, } \\
& \sum C_{2}=\sum_{p_{1} p_{2} \notin E\left(Q\left(G_{1}\right)\right)} \sum_{x_{1}, x_{2} \in V_{G_{2}}}\left[d\left(p_{1}, x_{1}\right) d\left(p_{2}, x_{2}\right)\right] \\
& p_{1} \in V\left(G_{1}\right) \\
& p_{2} \in V\left(Q\left(G_{1}\right)-V\left(G_{1}\right)\right) \\
& =\sum_{p_{1} p_{2} \notin E\left(Q\left(G_{1}\right)\right)} \sum_{x_{1}, x_{2} \in V_{G_{2}}}\left[\left(d_{G_{1}}\left(p_{1}\right)+d\left(x_{1}\right)\right) d_{Q\left(G_{1}\right)}\left(p_{2}\right)\right] \\
& p_{1} \in V\left(G_{1}\right) \\
& p_{2} \in V\left(Q\left(G_{1}\right)-V\left(G_{1}\right)\right) \\
& =\sum_{p_{1} p_{2} \notin E\left(Q\left(G_{1}\right)\right)} \sum_{x_{1}, x_{2} \in V_{G_{2}}}\left[d_{G_{1}}\left(p_{1}\right) d_{Q\left(G_{1}\right)}\left(p_{2}\right)+d\left(x_{1}\right) d_{Q\left(G_{1}\right)}\left(p_{2}\right)\right] \\
& p_{1} \in V\left(G_{1}\right) \\
& p_{2} \in V\left(Q\left(G_{1}\right)-V\left(G_{1}\right)\right)
\end{aligned}
$$

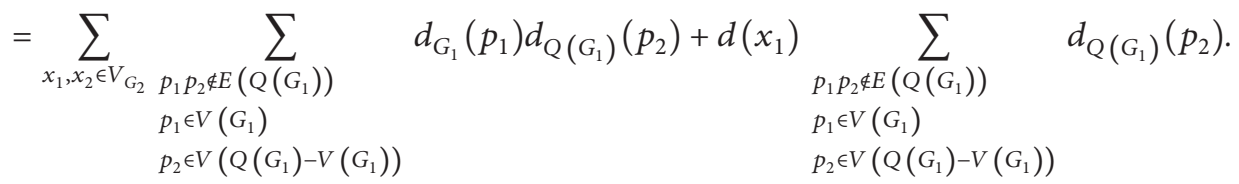


Note that

$$
\begin{aligned}
& \begin{aligned}
\bar{M}_{2}\left(G_{1}\right) \leq & \sum_{\substack{p_{1} p_{2} \notin E\left(Q\left(G_{1}\right)\right) \\
p_{1} \in V\left(G_{1}\right)}} d_{Q\left(G_{1}\right)}\left(p_{1}\right) \leq \bar{M}_{2} Q\left(G_{1}\right) \\
& p_{2} \in V\left(Q\left(G_{1}\right)-V\left(G_{1}\right)\right)
\end{aligned} \\
& 2 \bar{e}_{1} \leq \sum_{\substack{p_{1} p_{2} \notin E\left(Q\left(G_{1}\right)\right) \\
p_{1} \in V\left(G_{1}\right) \\
p_{2} \in V\left(Q\left(G_{1}\right)-V\left(G_{1}\right)\right)}} \sum d_{Q\left(G_{1}\right)}\left(p_{2}\right) \leq 2 \bar{e}_{Q\left(G_{1}\right)} \\
& n_{2}\left(n_{2}-1\right) \bar{M}_{2}\left(G_{1}\right)+4 e_{2}\left(n_{2}-1\right) \bar{e}_{1} \leq \sum C_{2} \leq n_{2}\left(n_{2}-1\right) \bar{M}_{2}\left(Q\left(G_{1}\right)\right)+4 e_{2}\left(n_{2}-1\right) \bar{e}_{Q}\left(G_{1}\right), \\
& \begin{aligned}
\sum C_{3}= & \sum_{\substack{p_{1} p_{2} \notin E\left(Q\left(G_{1}\right)\right) \\
p_{1} \in V\left(G_{1}\right)}} \sum_{x_{1}, x_{2} \in V_{G_{2}}}\left[d\left(p_{1}, x_{1}\right) d\left(p_{2}, x_{2}\right)\right] \\
& p_{2} \in V\left(Q\left(G_{1}\right)-V\left(G_{1}\right)\right)
\end{aligned} \\
& =\sum_{\substack{p_{1} p_{2} \notin E\left(Q\left(G_{1}\right)\right) \\
p_{1} \in V\left(G_{1}\right)}} \sum_{\substack{\left.x_{1}, x_{2} \in V_{G_{2}} \\
p_{2} \in V\left(G_{1}\right)-V\left(G_{1}\right)\right)}}\left[\left(d_{G_{1}}\left(p_{1}\right)+d\left(x_{1}\right)\right) d_{Q\left(G_{1}\right)}\left(p_{2}\right)\right] \\
& p_{2} \in V\left(Q\left(G_{1}\right)-V\left(G_{1}\right)\right) \\
& \begin{aligned}
&= \sum_{\substack{p_{1} p_{2} \notin E\left(Q\left(G_{1}\right)\right) \\
p_{1} \in V\left(G_{1}\right)}} \sum_{x_{1}, x_{2} \in V_{G_{2}}}\left[d_{G_{1}}\left(p_{1}\right) d_{Q\left(G_{1}\right)}\left(p_{2}\right)+d\left(x_{1}\right) d_{Q\left(G_{1}\right)}\left(p_{2}\right)\right] \\
& p_{2} \in V\left(Q\left(G_{1}\right)-V\left(G_{1}\right)\right)
\end{aligned}
\end{aligned}
$$

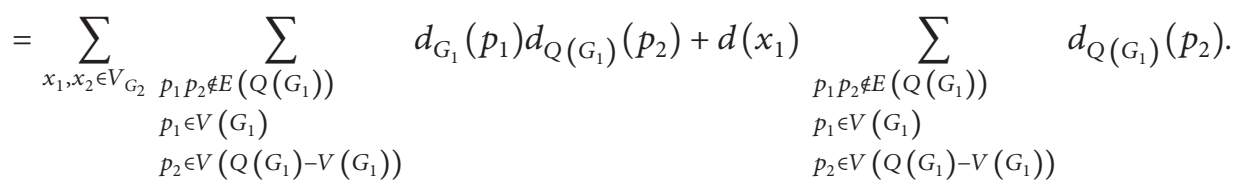


Note that

$$
\begin{aligned}
M_{2}\left(G_{1}\right) \leq & \sum_{\substack{p_{1} p_{2} \notin E\left(Q\left(G_{1}\right)\right) \\
p_{1} \in V\left(G_{1}\right) \\
p_{2} \in V\left(Q\left(G_{1}\right)-V\left(G_{1}\right)\right)}} d_{Q\left(G_{1}\right)}\left(p_{1}\right) \leq M_{2} Q\left(G_{1}\right), \\
2 e_{1} \leq & \sum_{\substack{p_{1} p_{2} \notin E\left(Q\left(G_{1}\right)\right) \\
p_{1} \in V\left(G_{1}\right) \\
p_{2} \in V\left(Q\left(G_{1}\right)-V\left(G_{1}\right)\right)}} d_{Q\left(G_{1}\right)}\left(p_{2}\right) \leq 2 e_{Q}\left(G_{1}\right), \\
n_{2}\left(n_{2}-1\right) M_{2}\left(G_{1}\right)+4 e_{2}\left(n_{2}-1\right) e_{1} \leq & \sum C_{3} \leq n_{2}\left(n_{2}-1\right) M_{2}\left(Q\left(G_{1}\right)\right)+4 e_{2}\left(n_{2}-1\right) e_{Q}\left(G_{1}\right) .
\end{aligned}
$$

Consequently,

$$
\begin{aligned}
& n_{2} \bar{M}_{2}\left(G_{1}\right)+4 e_{2} \bar{e}_{1}+n_{2}\left(n_{2}-1\right) \bar{M}_{2}\left(G_{1}\right)+4 e_{2}\left(n_{2}-1\right) \bar{e}_{1}+n_{2}\left(n_{2}-1\right) M_{2}\left(G_{1}\right)+4 e_{2}\left(n_{2}-1\right) e_{1} \\
& \leq \sum C \\
& \leq n_{2} \bar{M}_{2}\left(Q\left(G_{1}\right)\right)+4 e_{2} \bar{e}_{Q\left(G_{1}\right)}+n_{2}\left(n_{2}-1\right) \bar{M}_{2}\left(Q\left(G_{1}\right)\right)+4 e_{2}\left(n_{2}-1\right) \bar{e}_{Q\left(G_{1}\right)}+n_{2}\left(n_{2}-1\right) M_{2}\left(Q\left(G_{1}\right)\right)+4 e_{2}\left(n_{2}-1\right) e_{Q}\left(G_{1}\right)
\end{aligned}
$$

We obtained the required proof by putting the values of $\sum A+\sum B+\sum C$ in equation (25).

$$
\alpha_{1} \leq \bar{M}_{2}\left(G_{1+T} G_{2}\right) \leq \alpha_{2}
$$

Theorem 4. Let $G_{1}$ and $G_{2}$ be two graphs, then second Zagreb coindex of $G_{1+T} G_{2}$ is given as follows:

$$
\begin{aligned}
\alpha_{1}= & 4 e_{2}\left[\bar{e}_{1}+\left(n_{2}-1\right)\left(\bar{e}_{1}+e_{1}\right)\right]+2 n_{2}\left[\bar{M}_{2}\left(G_{1}\right)+\left(n_{2}-1\right)\left(\bar{M}_{2}\left(G_{1}\right)+M_{2}\left(G_{1}\right)\right)\right]+\left(n_{2}-1+\bar{e}_{2}\right)\left(M_{1}\left(G_{1}\right)+2 M_{2}\left(G_{1}\right)\right) \\
& +4 \overline{e_{2}} M_{1}\left(G_{1}\right)+4 e_{2} \bar{M}_{1}\left(G_{1}\right)+8\left(e_{2}+\overline{e_{2}}\right) M_{2}\left(G_{1}\right)+4\left(n_{2}+2\left(e_{2}+{\overline{e_{2}}}\right)\right) \bar{M}_{2}\left(G_{1}\right)+{\overline{e_{1}}}_{1} M_{1}\left(G_{2}\right) \\
& +4 e_{1} \bar{M}_{1}\left(G_{2}\right)+2\left(e_{1}+\overline{e_{1}}\right) M_{2}\left(G_{2}\right)+\left(n_{1}+2\left(e_{1}+\bar{e}_{1}\right)\right) \bar{M}_{2}\left(G_{2}\right)+2\left(M_{1}\left(G_{2}\right)+\bar{M}_{1}\left(G_{2}\right)\right)\left(M_{1}\left(G_{1}\right)+\bar{M}_{1}\left(G_{1}\right)\right), \\
\alpha_{2}= & 4 e_{2}\left[\bar{e}_{T\left(G_{1}\right)}+\left(n_{2}-1\right)\left(\bar{e}_{T\left(G_{1}\right)}+e_{T\left(G_{1}\right)}\right)\right]+2 n_{2}\left(\bar{M}_{2}\left(T\left(G_{1}\right)\right)\right)+\left(n_{2}-1\right)\left(M_{2}\left(T\left(G_{1}\right)\right)+\bar{M}_{2}\left(T\left(G_{1}\right)\right)\right), \\
\alpha_{2}= & 4 e_{2}\left[\bar{e}_{T\left(G_{1}\right)}+\left(n_{2}-1\right)\left(\bar{e}_{T\left(G_{1}\right)}+e_{T}\left(G_{1}\right)\right)\right]+2 n_{2}\left(\bar{M}_{2}\left(T\left(G_{1}\right)\right)\right)+\left(n_{2}-1\right)\left(M_{2}\left(T\left(G_{1}\right)\right)+\bar{M}_{2}\left(T\left(G_{1}\right)\right)\right) \\
& +8\left(e_{2}+\overline{e_{2}}\right) M_{2}\left(G_{1}\right)+4\left(n_{2}+2\left(e_{2}+{\overline{e_{2}}}_{2}\right) \bar{M}_{2}\left(G_{1}\right)+\overline{e_{1}} M_{1}\left(G_{2}\right)+4 e_{1} \bar{M}_{1}\left(G_{2}\right)+2\left(e_{1}+\overline{e_{1}}\right) M_{2}\left(G_{2}\right)\right. \\
& +\left(n_{1}+2\left(e_{1}+\overline{e_{1}}\right)\right) \bar{M}_{2}\left(G_{2}\right)+2\left(M_{1}\left(G_{2}\right)+\bar{M}_{1}\left(G_{2}\right)\right)\left(M_{1}\left(G_{1}\right)+\bar{M}_{1}\left(G_{1}\right)\right) .
\end{aligned}
$$

Proof. Using equation (2), we have

$$
\bar{M}_{2}\left(G_{1+T} G_{2}\right)=\sum_{\left(p_{1}, p_{2}\right)} \sum_{\left(q_{1}, q_{2}\right) \notin E\left(G_{1+T} G_{2}\right)}\left[d\left(p_{1}, q_{1}\right) d\left(p_{2}, q_{2}\right)\right]=\sum A+\sum B+\sum C .
$$


Using equation (40), we directly have

$$
\begin{aligned}
& 2 \bar{e}_{2} M_{2}\left(G_{1}\right)+2\left(n_{2}-1\right) M_{2}\left(G_{1}\right)+\left(n_{2}-1\right) M_{1}\left(G_{1}\right)+\bar{e}_{2} M_{1}\left(G_{1}\right) \\
& \leq \sum A \leq n_{2} \bar{M}_{2}\left(T\left(G_{1}\right)\right)+\left(n_{2}-1\right) M_{1}\left(T\left(G_{1}\right)\right) \\
& \quad+\bar{e}_{2} M_{1}\left(T\left(G_{1}\right)\right)+2\left(n_{2}-1\right) M_{2}\left(T\left(G_{1}\right)\right)+2 \bar{e}_{2} M_{2}\left(T\left(G_{1}\right)\right)+2\left(n_{2}-1\right) \bar{M}_{2}\left(T\left(G_{1}\right)\right)+2 \bar{e}_{2} \bar{M}_{2}\left(T\left(G_{1}\right)\right) .
\end{aligned}
$$

Using equation (15), we directly have

$$
\begin{aligned}
& \sum B=4 \overline{e_{2}} M_{1}\left(G_{1}\right)+4 e_{2} \bar{M}_{1}\left(G_{1}\right)+8\left(e_{2}+\overline{e_{2}}\right) M_{2}\left(G_{1}\right)+4 n_{2}+2\left(e_{2}+\overline{e_{2}}\right) \bar{M}_{2}\left(G_{1}\right) \\
& +\overline{e_{1}} M_{1}\left(G_{2}\right)+4 e_{1} \bar{M}_{1}\left(G_{2}\right) \\
& +2\left(e_{1}+\overline{e_{1}}\right) M_{2}\left(G_{2}\right)+\left(n_{1}+2\left(e_{1}+\overline{e_{1}}\right)\right) \bar{M}_{2}\left(G_{2}\right)+2\left(M_{1}\left(G_{2}\right)\right. \\
& \left.+\bar{M}_{1}\left(G_{2}\right)\right)\left(M_{1}\left(G_{1}\right)+\bar{M}_{1}\left(G_{1}\right)\right) \text {, } \\
& \sum C=\sum C_{1}+\sum C_{2}+\sum C_{3} \\
& \sum C_{1}=\sum_{t_{1} t_{2} \notin E\left(T\left(G_{1}\right)\right)} \sum_{q \in V_{G_{2}}}\left[d\left(p_{1}, q\right) d\left(p_{2}, q\right)\right] \\
& p_{1} \in V\left(G_{1}\right) \\
& p_{2} \in V\left(T\left(G_{1}\right)-G_{1}\right) \\
& 2 n_{2} \bar{M}_{2}\left(G_{1}\right)+4 e_{2} \bar{e}_{1} \leq \sum C_{1} \leq 2 n_{2} \bar{M}_{2}\left(T\left(G_{1}\right)\right)+4 e_{2} \bar{e}_{T\left(G_{1}\right)} \\
& \sum C_{2}=\sum_{p_{1} p_{2} \notin E\left(T\left(G_{1}\right)\right)} \sum_{q_{1}, q_{2} \in V_{G_{2}}}\left[d\left(p_{1}, q_{1}\right) d\left(p_{2}, q_{2}\right)\right] \\
& p_{1} \in V\left(G_{1}\right) \\
& p_{2} \in V\left(T\left(G_{1}\right)-V\left(G_{1}\right)\right) \\
& 2 n_{2}\left(n_{2}-1\right) \bar{M}_{2}\left(G_{1}\right)+4 e_{2}\left(n_{2}-1\right) \bar{e}_{1} \leq \sum C_{2} \leq 2 n_{2}\left(n_{2}-1\right) \bar{M}_{2}\left(T\left(G_{1}\right)\right)+4 e_{2}\left(n_{2}-1\right) \bar{e}_{T\left(G_{1}\right)}, \\
& \sum C_{3}=\sum_{p_{1} p_{2} \in E\left(T\left(G_{1}\right)\right)} \sum_{q_{1}, q_{2} \in V_{G_{2}}}\left[d\left(p_{1}, q_{1}\right) d\left(p_{2}, q_{2}\right)\right] \\
& p_{1} \in V\left(G_{1}\right) \\
& p_{2} \in V\left(T\left(G_{1}\right)-V\left(G_{1}\right)\right) \\
& 2 n_{2}\left(n_{2}-1\right) M_{2}\left(G_{1}\right)+4 e_{2}\left(n_{2}-1\right) e_{1} \leq \sum C_{3} \leq 2 n_{2}\left(n_{2}-1\right) M_{2}\left(T\left(G_{1}\right)\right)+4 e_{2}\left(n_{2}-1\right) e_{T\left(G_{1}\right)}
\end{aligned}
$$

\section{Consequently,}

$2 n_{2} \bar{M}_{2}\left(G_{1}\right)+4 e_{2} \bar{e}_{1}+2 n_{2}\left(n_{2}-1\right) \bar{M}_{2}\left(G_{1}\right)+4 e_{2}\left(n_{2}-1\right) \bar{e}_{1}+2 n_{2}\left(n_{2}-1\right) M_{2}\left(G_{1}\right)+4 e_{2}\left(n_{2}-1\right) e_{1}$

$\leq \sum C$

$\leq 2 n_{2} \bar{M}_{2}\left(T\left(G_{1}\right)\right)+4 e_{2} \bar{e}_{T\left(G_{1}\right)}+2 n_{2}\left(n_{2}-1\right) \bar{M}_{2}\left(T\left(G_{1}\right)\right)+4 e_{2}\left(n_{2}-1\right) \bar{e}_{T\left(G_{1}\right)}+2 n_{2}\left(n_{2}-1\right) M_{2}\left(T\left(G_{1}\right)\right)+4 e_{2}\left(n_{2}-1\right) e_{T\left(G_{1}\right)}$. 
TABLE 1: Exact and bounded values of certain $F$-sum graphs.

\begin{tabular}{lccc}
\hline F-sum operation & Lower bounds & Exact values & Upper bounds \\
\hline $\bar{M}_{2}\left(G_{1+S} G_{2}\right)$ & 152 & 160 & 312 \\
$\bar{M}_{2}\left(G_{1+R} G_{2}\right)$ & 216 & 232 & 728 \\
$\bar{M}_{2}\left(G_{1+Q} G_{2}\right)$ & 106 & 220 & 338 \\
$\bar{M}_{2}\left(G_{1+T} G_{2}\right)$ & 150 & 300 & 642
\end{tabular}

We obtained required results by putting the values of $\sum A+\sum B+\sum C$ in equation (48).

\section{Conclusion}

In this paper, we have computed second Zagreb coindex of $F$-sum graphs such as $\bar{M}_{2}\left(G_{1+S} G_{2}\right), \bar{M}_{2}\left(G_{1+R} G_{2}\right)$, $\bar{M}_{2}\left(G_{1+Q} G_{2}\right)$, and $\bar{M}_{2}\left(G_{1+T} G_{2}\right)$. The obtained results are illustrated with the help of specific class graphs of $F$-sum graphs. Let $G_{1} \cong P_{3}$ and $G_{2} \cong P_{2}$, then the lower and upper bounds of first Zagreb coindex for their F-sum graph are given in Table 1.

Now, we close our discussion that the problem is still open to compute the other generalized coindices (first general Zagreb and general Randic coindices) for the F-sum graphs.

\section{Data Availability}

The data used to support this study are included within the article. However, the reader may request the corresponding author for more details of the data.

\section{Conflicts of Interest}

The authors declare that they have no conflicts of interest.

\section{References}

[1] G. Rücker and C. Rücker, "On topological indices, boiling points, and cycloalkanes," Journal of Chemical Information and Computer Sciences, vol. 39, no. 5, pp. 788-802, 1999.

[2] A. R. Matamala and E. Estrada, "Generalised topological indices: optimisation methodology and physico-chemical interpretation," Chemical Physics Letters, vol. 410, no. 4-6, pp. 343-347, 2005.

[3] H. Gonzalez-Diaz, S. Vilar, L. Santana, and E. Uriarte, "Medicinal chemistry and bioinformatics - current trends in drugs discovery with networks topological indices," Current Topics in Medicinal Chemistry, vol. 7, no. 10, pp. 1015-1029, 2007.

[4] W. Yan, B.-Y. Yang, and Y.-N. Yeh, "The behavior of Wiener indices and polynomials of graphs under five graph decorations," Applied Mathematics Letters, vol. 20, no. 3, pp. 290-295, 2007.

[5] M. V. Diudea, QSPR/QSAR Studies by Molecular Descriptors, Nova ccience Publishers, Hauppauge, NY, USA, 2001.

[6] I. Gutman, "Degree-based topological indices," Croatica Chemica Acta, vol. 86, no. 4, pp. 351-361, 2013.

[7] H. Wiener, "Structural determination of paraffin boiling points," Journal of the American Chemical Society, vol. 69, no. 1, pp. 17-20, 1947.
[8] I. Gutman and N. Trinajstić, "Graph theory and molecular orbitals. Total $\varphi$-electron energy of alternant hydrocarbons," Chemical Physics Letters, vol. 17, no. 4, pp. 535-538, 1972.

[9] K. C. Das and I. Gutman, "Some properties of the second Zagreb index," Communications in Mathematical and in Computer Chemistry, vol. 52, no. 1, pp. 1-3, 2004.

[10] Z. Yan, H. Liu, and H. Liu, "Sharp bounds for the second Zagreb index of unicyclic graphs," Journal of Mathematical Chemistry, vol. 42, no. 3, pp. 565-574, 2007.

[11] M. Ahmad, M. Saeed, M. Javaid, and M. Hussain, "Exact formula and improved bounds for general sum-connectivity index of graph-operations," IEEE Access, vol. 7, pp. 167290167299, 2019.

[12] A. R. Ashrafi, T. Došlić, and A. Hamzeh, "The Zagreb coindices of graph operations," Discrete Applied Mathematics, vol. 158, no. 15, pp. 1571-1578, 2010.

[13] A. R. Ashrafi, T. Došlic, and A. Hamzeh, "Extremal graphs with respect to the Zagreb coindices," MATCH Communications in Mathematical and in Computer Chemistry, vol. 65, no. 1, pp. 85-92, 2011.

[14] K. C. Das, N. Akgunes, M. Togan, A. Yurttas, I. N. Cangul, and A. S. Cevik, "On the first Zagreb index and multiplicative Zagreb coindices of graphs," Analele Universitatii "Ovidius" Constanta - Seria Matematica, vol. 24, no. 1, pp. 153-176, 2016.

[15] I. Gutman, "On coindices of graphs and their complements," Applied Mathematics and Computation, vol. 305, pp. 161-165, 2017.

[16] N. De, S. M. A. Nayeem, and A. Pal, "The F-coindex of some graph operations,” Springer, vol. 5, no. 1, pp. 1-13, 2016.

[17] M. Javaid, U. Ali, and J. B. Liu, "Computing analysis for first Zagreb connection index and coindex of resultant graphs," Mathematical Problems in Engineering, vol. 2021, Article ID 6019517, 19 pages, 2021.

[18] S. H. Ramane and Y. Saroja, "Talwar and ismail naci Cangul, "Transmission and reciprocal transmission based topological Co-indices of graphs," European Journal of Pure and Applied Mathematics, vol. 5, pp. 1057-1071, 2020.

[19] T. Mansour and C. Song, "The a and ( $a, b)$ analogs of zagreb indices and coindices of graphs," International Journal of Combinatorics, vol. 2012, Article ID 909285, 10 pages, 2012.

[20] C. M. Da Fonseca and D. Stevanovic, "Further properties of the second Zagreb index," Communications in Mathematical and in Computer Chemistry, vol. 72, pp. 655-668, 2014.

[21] M. Eliasi and B. Taeri, "Four new sums of graphs and their Wiener indices," Discrete Applied Mathematics, vol. 157, no. 4, pp. 794-803, 2009.

[22] H. Deng, D. Sarala, S. K. Ayyaswamy, and S. Balachandran, "The Zagreb indices of four operations on graphs," Applied Mathematics and Computation, vol. 275, pp. 422-431, 2016.

[23] S. Akhter and M. Imran, "Computing the forgotten topological index of four operations on graphs," AKCE International Journal of Graphs and Combinatorics, vol. 14, no. 1, pp. 70-79, 2017.

[24] J.-B. Liu, S. Javed, M. Javaid, and K. Shabbir, "Computing first general Zagreb index of operations on graphs," IEEE Access, vol. 7, pp. 47494-47502, 2019.

[25] A. M. Alanazi, F. Farid, M. Javaid, and A. Munagi, "Computing exact values for gutman indices of sum graphs under cartesian product," Mathematical Problems in Engineering, vol. 2021, Article ID 5569997, 20 pages, 2021. 